\title{
Cyclometallation Geometry of the Bridging Ligand as a Tuning Tool for Photophysics of Dinuclear Ir(III) Complexes
}

\author{
Marsel Z. Shafikov*,+, Andrey V. Zaytsev ${ }^{\S}$, Valery N. Kozhevnikov*, \\ ${ }^{\dagger}$ Institut für Physikalische und Theoretische Chemie, Universität Regensburg, Universitätsstrasse 31, \\ Regensburg, D-93053, Germany. \\ $\$$ Department for Technology of Organic Synthesis, Institute of Chemical Technology, Ural Federal \\ University, Mira 19, Ekaterinburg, 620002, Russia \\ ${ }^{\S}$ Department of Applied Sciences, Northumbria University, Newcastle upon Tyne, NE1 8ST, U.K.
}

\section{Synthesis and chemical characterization.}

\section{General}

All solvents and reagents were purchased from commercial suppliers and used without further purification. NMR spectra were recorded on a JEOL ECS400FT Delta spectrometer $(399.78 \mathrm{MHz}$ for $1 \mathrm{H} \mathrm{NMR})$. Chemical shifts are reported in parts per million ( $\mathrm{ppm}$ ) relative to a tetramethylsilane internal standard. Elemental analysis was carried out on ELEMENTAR vario MICRO CUBE instrument at central analytical services of the University of Regensburg. FieldDesorption Mass-spectroscopy (FD-MS) was performed on a JEOL AccuTOF GCX instrument at the central analytical services of the University of Regensburg. Intermediate dicholoro-bridged complex 4 was prepared as described previously ${ }^{1}$.

\section{Synthetic procedures and characterizations}

Two proligands 5 and $\mathbf{6}$ were prepared by the reaction of two molar equivalents of the corresponding aldehydes $\mathbf{2}$ and $\mathbf{3}$ with one molar equivalent of dithoxamide $\mathbf{1}$ in presence of an oxidative agent chloranil (Scheme 1). The proligands were reacted with a well-known ${ }^{1}$ intermediate di-chlorobridged Ir(III) complex $\mathbf{4}$ in boiling toluene in presence of silver triflate as a chloride scavenger. 


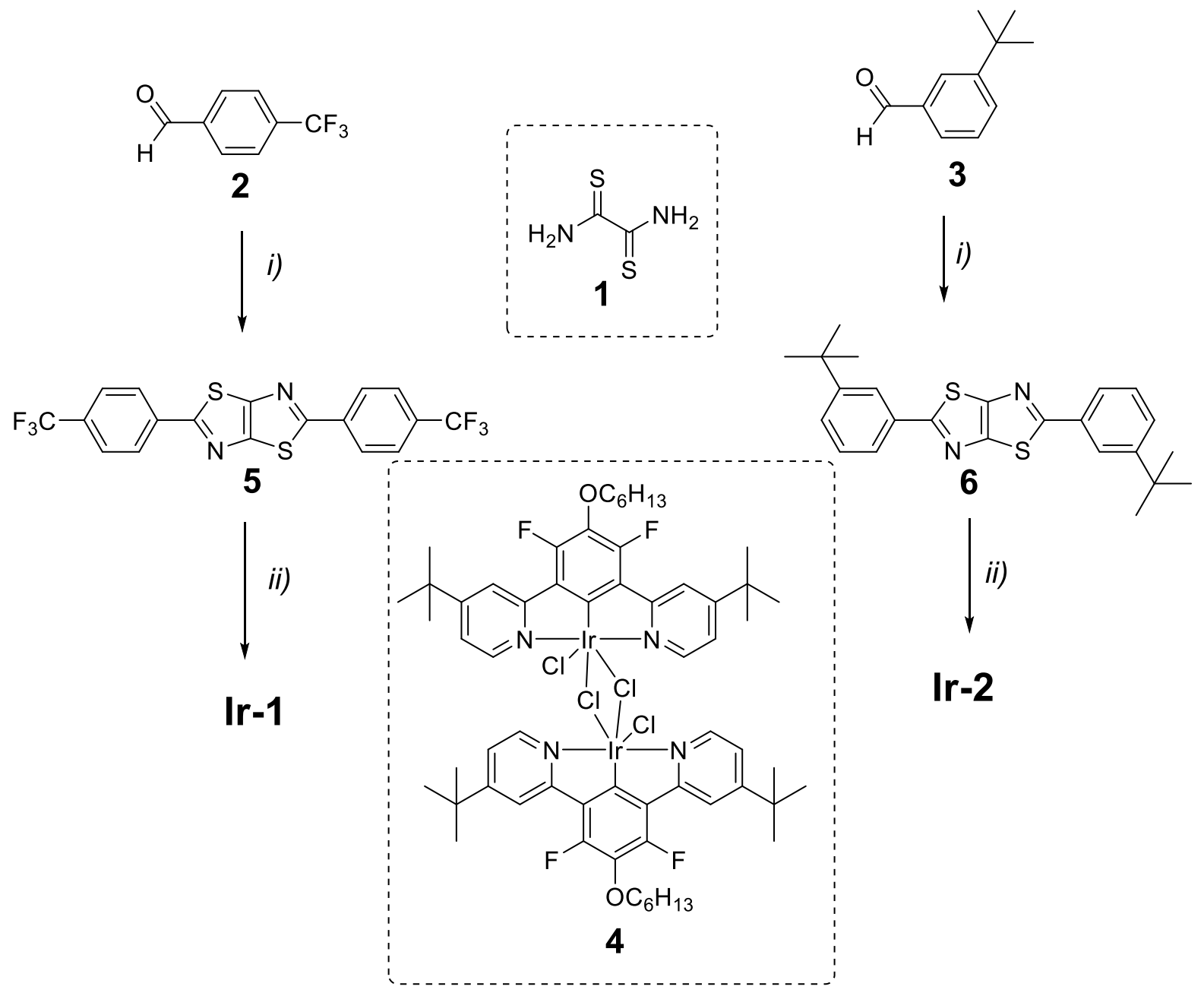

Scheme S1. Reaction conditions: i) a) dithiooxamide 1 (0.5 eq), nitrobenzene, $\mathrm{MW}, 180^{\circ} \mathrm{C}, 30$ min b) chloranil, THF, reflux, 10 min; 55\% for 5, 39\% for 6; ii) complex 4, AgOTf, toluene, reflux, 16 hours; Ir-1 79\%, Ir-2 75\%.

Proligand 5.

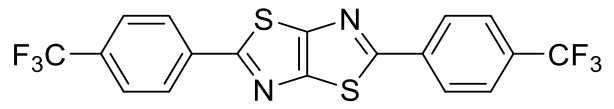

4-Trifluoromethylbenzaldehyde ( $697 \mathrm{mg}, 4 \mathrm{mmol}, 2$ eq.) and dithiooxamide ( $240 \mathrm{mg}, 2 \mathrm{mmol}, 1 \mathrm{eq}$.) were mixed together in nitrobenzene $(2 \mathrm{~mL})$ and was irradiated with microwaves to maintain temperature of the reaction mixture at $180^{\circ} \mathrm{C}$ for $30 \mathrm{~min}$. The reaction mixture was transferred into a round-bottom flask containing chloranil ( $246 \mathrm{mg}, 1 \mathrm{mmol}, 0.5 \mathrm{eq}$.) in THF (10 mL) and the content was heated to reflux for 10 $\mathrm{min}$. The reaction mixture was allowed to cool to room temperature, $\mathrm{MeOH}(20 \mathrm{~mL})$ was added and the suspension was cooled to $0^{\circ} \mathrm{C}$ using an ice-water bath. The resulting precipitate was filtered washed with MeOH followed by THF to give proligand $5(500 \mathrm{mg}, 59 \%)$ as a beige solid. ${ }^{2}$

${ }^{1} \mathrm{H}$ NMR (400 MHz, $\left.\mathrm{CDCl}_{3}\right): \delta 8.13(\mathrm{~d}, 4 \mathrm{H}, J=8.3 \mathrm{~Hz}, \mathrm{Ar}-\mathrm{H}), 7.75(\mathrm{~d}, 4 \mathrm{H}, J=8.3 \mathrm{~Hz}, \mathrm{Ar}-\mathrm{H})$.

Proligand 6. 


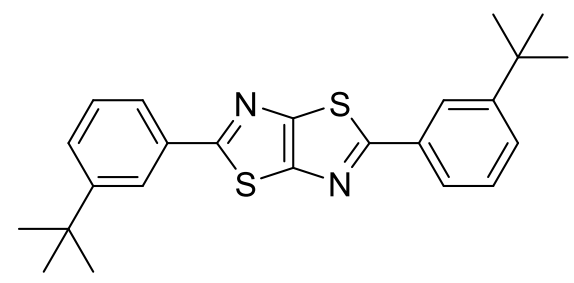

Prepared as 5 using 3-tert-butylbenzaldehyde (1.15 g, $7.08 \mathrm{mmol})$, dithiooxamide (426 mg, 3.54 mmol), nitrobenzene ( $3.5 \mathrm{~mL})$, chloranil $(435 \mathrm{mg}, 1.77 \mathrm{mmol})$ and THF (20 $\mathrm{mL})$ to give proligand $6(555 \mathrm{mg}, 39 \%)$ as mustard-coloured solid.

${ }^{1} \mathrm{H}$ NMR (400 MHz, CDCl $): \delta 8.06$ (t, 2H, $\left.J=1.9 \mathrm{~Hz}, \mathrm{Ar}-\mathrm{H}\right), 7.78$ (d, 2H, J=7.7 Hz, Ar-H), 7.51 (app.d, 2H, $J=8.4 \mathrm{~Hz}, \mathrm{Ar}-\mathrm{H}$ ), 7.43 (app. t, $2 \mathrm{H}, J=7.7 \mathrm{~Hz}, \mathrm{Ar}-\mathrm{H}), 1.41\left(\mathrm{~s}, 18 \mathrm{H}, 2 \times \mathrm{C}\left(\mathrm{CH}_{3}\right)_{3}\right)$

${ }^{13} \mathrm{C} \mathrm{NMR}$ (100 MHz, $\mathrm{CDCl}_{3}$ ): $\delta 169.8$ (quat.), 152.4 (quat.), 150.9 (quat.), 133.9 (quat.), 129.0 $(\mathrm{CH}), 128.1(\mathrm{CH}), 124.0(\mathrm{CH}), 123.4(\mathrm{CH}), 35.1$ (quat., $\left.C\left(\mathrm{CH}_{3}\right)_{3}\right), 31.4\left(\mathrm{C}\left(\mathrm{CH}_{3}\right)_{3}\right)$.

\section{Complex Ir-1.}

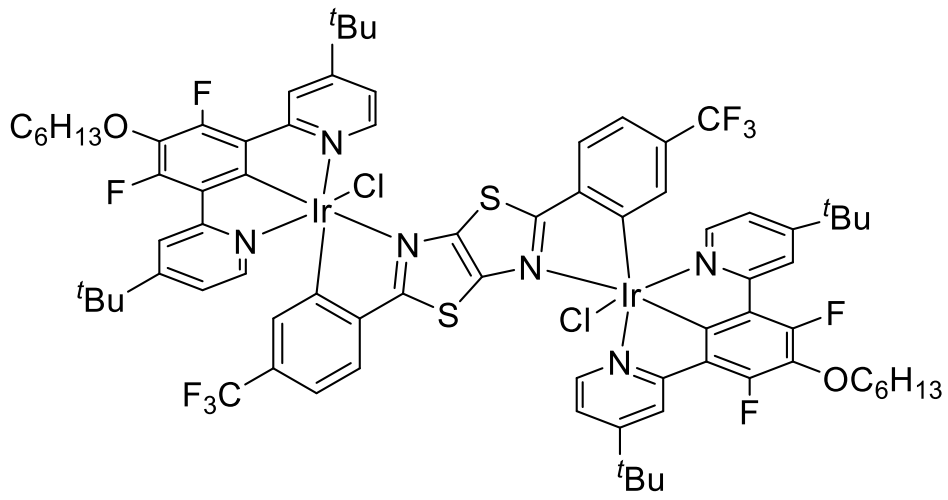

The dichlorobridged Ir(III) complex 4 (160 mg, $108 \mu \mathrm{mol}, 1$ eq.) and proligand 5 (46 mg, 108 $\mu \mathrm{mol}, 1$ eq.) were suspended in toluene $(20 \mathrm{~mL}), \operatorname{AgOTf}(0.1 \mathrm{M}$ in toluene) $(3.24 \mathrm{~mL}, 324 \mu \mathrm{mol}$, 3 eq.) was added. The reaction mixture was heated to reflux under Ar atmosphere for 16 hours. The content of the flask was cooled to about $60^{\circ} \mathrm{C}$ and $2 \mathrm{M} \mathrm{HCl}(5.0 \mathrm{~mL})$ was added. The volatiles were removed under reduced pressure. The solid was suspended in DCM (20 mL), dried over $\mathrm{MgSO}_{4}$ and filtered through a plug of Celite washing with DCM until the DCM washings ran colourless. The combined DCM washings were evaporated; and the residue was subjected to flash column chromatography using as an eluent to give Ir-1 (157 mg, 79\%) as a light brown solid.

${ }^{1} \mathrm{H}$ NMR $\left(400 \mathrm{MHz}, \mathrm{CDCl}_{3}\right.$ ): $\delta 8.18$ (s, 4H, Ar-H), 7.69 (app.d, 6H, Ar-H), 7,02-6.99 (m, 6H, ArH), 6.37 (s, 2H, Ar-H), 4.24 (app.t, 4H, $2 \times \mathrm{OCH}_{2}$ ), 1.93 (app. quin, 4H, $2 \times \mathrm{CH}_{2}$ ), 1.62 (app quin, $\left.4 \mathrm{H}, 2 \times \mathrm{CH}_{2}\right), 1.46-1.42\left(\mathrm{~m}, 8 \mathrm{H}, 4 \times \mathrm{CH}_{2}\right), 1.36\left(\mathrm{~s}, 36 \mathrm{H}, 4 \times \mathrm{C}\left(\mathrm{CH}_{3}\right)_{3}\right), 0.97$ (app. t, $6 \mathrm{H}, 2 \times$ $\left.\mathrm{CH}_{2} \mathrm{CH}_{3}\right)$. 
${ }^{13} \mathrm{C} \mathrm{NMR}\left(100 \mathrm{MHz}, \mathrm{CDCl}_{3}\right): \delta 180.1,169.5,165.6,162.4,156.7,154.1,150.9(\mathrm{CH}), 148.4,146.8$, 144.1, $132.1(\mathrm{CH}), 131.3,130.6,124.9(\mathrm{CH}), 124.1,120.9(\mathrm{CH}), 120.6(\mathrm{CH}), 119.4(\mathrm{CH}), 75.7$ $\left(\mathrm{OCH}_{2}\right), 35.3$ (quat., $\left.C\left(\mathrm{CH}_{3}\right)_{3}\right), 31.8\left(\mathrm{CH}_{2}\right), 30.6\left(\mathrm{C}\left(\mathrm{CH}_{3}\right)_{3}\right), 30.3\left(\mathrm{CH}_{2}\right), 25.7\left(\mathrm{CH}_{2}\right), 22.8\left(\mathrm{CH}_{2}\right)$, $14.3\left(\mathrm{CH}_{2} \mathrm{CH}_{3}\right)$.

${ }^{19} \mathrm{~F}$ NMR (376 MHz, $\left.\mathrm{CDCl}_{3}\right)$-125.22 (Ar-F), -62.64 $\left(\mathrm{CF}_{3}\right)$.

Field desorption mass-spectrometry (FD-MS): calculated for $[\mathrm{M}]^{+}\left(\mathrm{C}_{78} \mathrm{H}_{80} \mathrm{Cl}_{2} \mathrm{~F}_{10} \mathrm{Ir}_{2} \mathrm{~N}_{6} \mathrm{O}_{2} \mathrm{~S}_{2}\right)$ 1842.43, found 1842.3870 .

Elemental analysis. Calculated for $\mathrm{C}_{78} \mathrm{H}_{80} \mathrm{Cl}_{2} \mathrm{~F}_{10} \mathrm{Ir}_{2} \mathrm{~N}_{6} \mathrm{O}_{2} \mathrm{~S}_{2}$ : C 50.83, H 4.38, N $4.56 \%$; found C $50.74, \mathrm{H} 4.42, \mathrm{~N} 4.41 \%$.

Synthesis of (Ir-2).

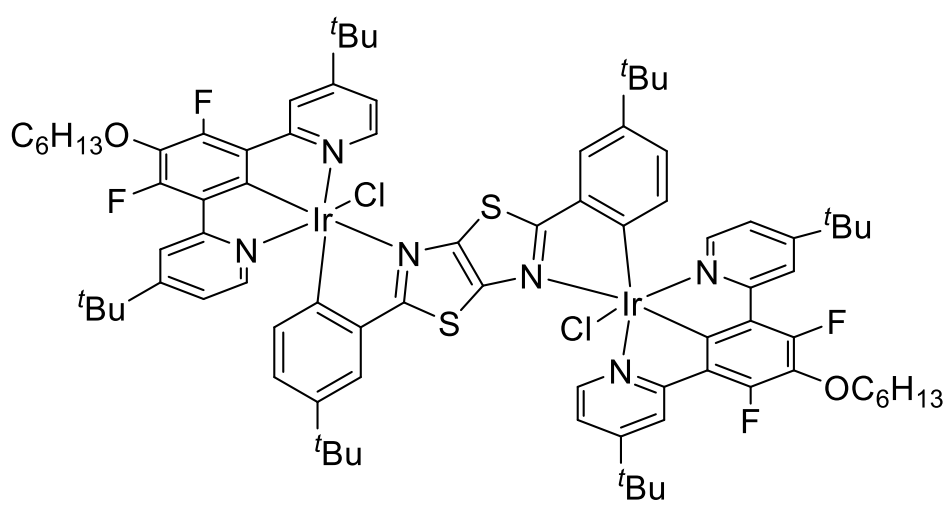

The dichlorobridged Ir(III) complex 4 (150 mg, $101 \mu \mathrm{mol}, 1$ eq.) and proligand 6 (41 mg, 101 $\mu \mathrm{mol}, 1$ eq.) were suspended in toluene (20 mL), $\operatorname{AgOTf}(0.1 \mathrm{M}$ in toluene) (3.03 mL, $303 \mu \mathrm{mol}$, 3 eq.) was added. The reaction mixture was heated to reflux under Ar atmosphere for 16 hours. The content of the flask was cooled to about $60^{\circ} \mathrm{C}$ and $2 \mathrm{M} \mathrm{HCl}(4.5 \mathrm{~mL})$ was added. The volatiles were removed under reduced pressure. The solid was suspended in DCM (20 mL), dried over $\mathrm{MgSO}_{4}$ and filtered through a plug of Celite washing with DCM until the DCM washings ran colourless. The combined DCM washings were evaporated; and the residue was subjected to flash column chromatography using as an eluent to give Ir-2 (138 mg, 75\%) as a light brown solid.

${ }^{1} \mathrm{H}$ NMR (400 MHz, $\mathrm{CD}_{2} \mathrm{Cl}_{2}$ ): $\delta 8.21$ (d, 4H, $\left.J=2.1 \mathrm{~Hz}, \mathrm{Ar}-\mathrm{H}\right), 7.88(\mathrm{~d}, 4 \mathrm{H}, J=6.6 \mathrm{~Hz}, \mathrm{Ar}-\mathrm{H}) 7.63$ $(\mathrm{d}, 2 \mathrm{H}, J=2.1 \mathrm{~Hz}, \mathrm{Ar}-\mathrm{H}), 7.06(\mathrm{dd}, 4 \mathrm{H}, J=6.1$ and $2.1 \mathrm{~Hz}, \mathrm{Ar}-\mathrm{H}), 6.71(\mathrm{dd}, 2 \mathrm{H}, J=8.2$ and 2.1 $\mathrm{Hz}, \mathrm{Ar}-\mathrm{H}), 6.02$ (d, 2H, $J=8.1 \mathrm{~Hz}, \mathrm{Ar}-\mathrm{H}), 4.26$ (t, 4H, $\left.J=6.7 \mathrm{~Hz}, 2 \times \mathrm{OCH}_{2}\right), 1.93$ (app.p, 4H, 2 $\left.\times \mathrm{CH}_{2}\right), 1.65-1.58\left(\mathrm{~m}, 4 \mathrm{H}, 2 \times \mathrm{CH}_{2}\right), 1.45-1.42\left(\mathrm{~m}, 6 \mathrm{H}, 3 \times \mathrm{CH}_{2}\right), 1.41\left(\mathrm{~s}, 36 \mathrm{H}, 4 \times \mathrm{C}\left(\mathrm{CH}_{3}\right)_{3}\right)$, $1.16\left(\mathrm{~s}, 18 \mathrm{H}, 2 \times \mathrm{C}\left(\mathrm{CH}_{3}\right)_{3}\right), 0.98\left(\mathrm{t}, 6 \mathrm{H}, J=7.0 \mathrm{~Hz}, 2 \times \mathrm{CH}_{2} \mathrm{CH}_{3}\right)$

${ }^{13} \mathrm{C}$ NMR (100 MHz, $\left.\mathrm{CD}_{2} \mathrm{Cl}_{2}\right): \delta 181.2,171.9,165.6,162.5,156.9,154.3,151.7(\mathrm{CH}), 147.7$, 145.6, 142.2, 141.1, $135.7(\mathrm{CH}), 130.3,128.4(\mathrm{CH}), 124.3,122.3(\mathrm{CH}), 120.7(\mathrm{CH}), 76.0\left(\mathrm{OCH}_{2}\right)$, 
35.4 (quat., $\left.C\left(\mathrm{CH}_{3}\right)_{3}\right), 34.3$ (quat., $\left.\left.\left.C\left(\mathrm{CH}_{3}\right)_{3}\right), 32.1\left(\mathrm{CH}_{2}\right), 31.4\left(\mathrm{C}_{(\mathrm{CH}}\right)_{3}\right)_{3}\right), 30.6\left(\mathrm{C}_{\left.\left(\mathrm{CH}_{3}\right)_{3}\right), 30.5}\right.$ $\left(\mathrm{CH}_{2}\right), 26.0\left(\mathrm{CH}_{2}\right), 23.1\left(\mathrm{CH}_{2}\right), 14.3\left(\mathrm{CH}_{2} \mathrm{CH}_{3}\right)$.

${ }^{19}$ F NMR (376 MHz, $\left.\mathrm{CD}_{2} \mathrm{Cl}_{2}\right):-126.15$

Field desorption mass-spectrometry (FD-MS): calculated for $[\mathrm{M}]^{+}\left(\mathrm{C}_{84} \mathrm{H}_{98} \mathrm{Cl}_{2} \mathrm{~F}_{4} \mathrm{Ir}_{2} \mathrm{~N}_{6} \mathrm{O}_{2} \mathrm{~S}_{2}\right)$ 1818.58, found 1818.5171 .

Elemental analysis. Calculated for $\mathrm{C}_{84} \mathrm{H}_{98} \mathrm{Cl}_{2} \mathrm{~F}_{4} \mathrm{Ir}_{2} \mathrm{~N}_{6} \mathrm{O}_{2} \mathrm{~S}_{2}$ : C 55.46, H 5.43, N $4.62 \%$; found C $55.13, \mathrm{H} 5.63, \mathrm{~N} 4.62 \%$. 
AVZ-VK1-50-1
single_pulse
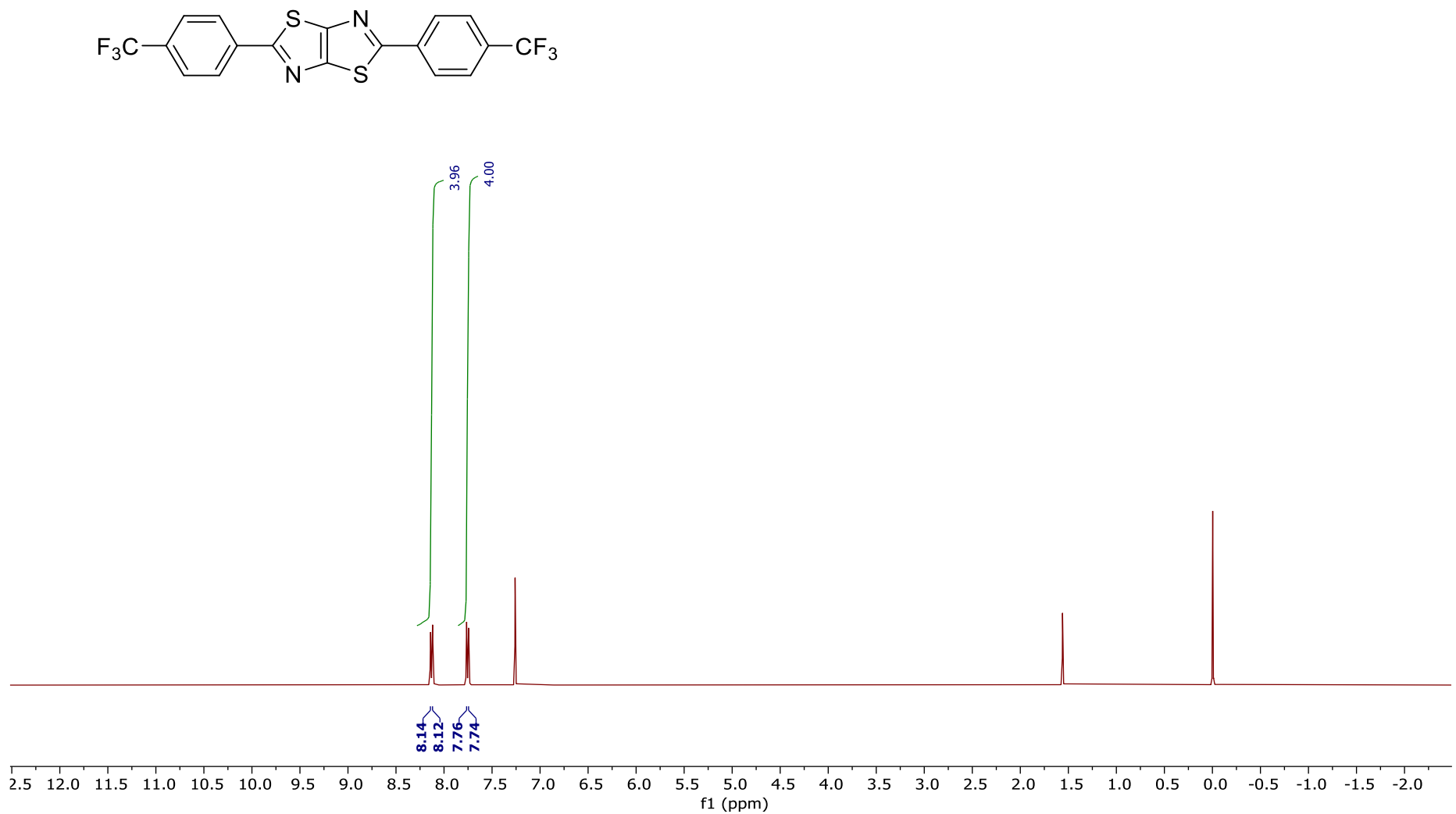

Figure S1. ${ }^{1} \mathrm{H}$ NMR spectrum of proligand 5 in $\mathrm{CDCl}_{3}$ 
AVZ-VK2-43-1
single pulse

single_pulse

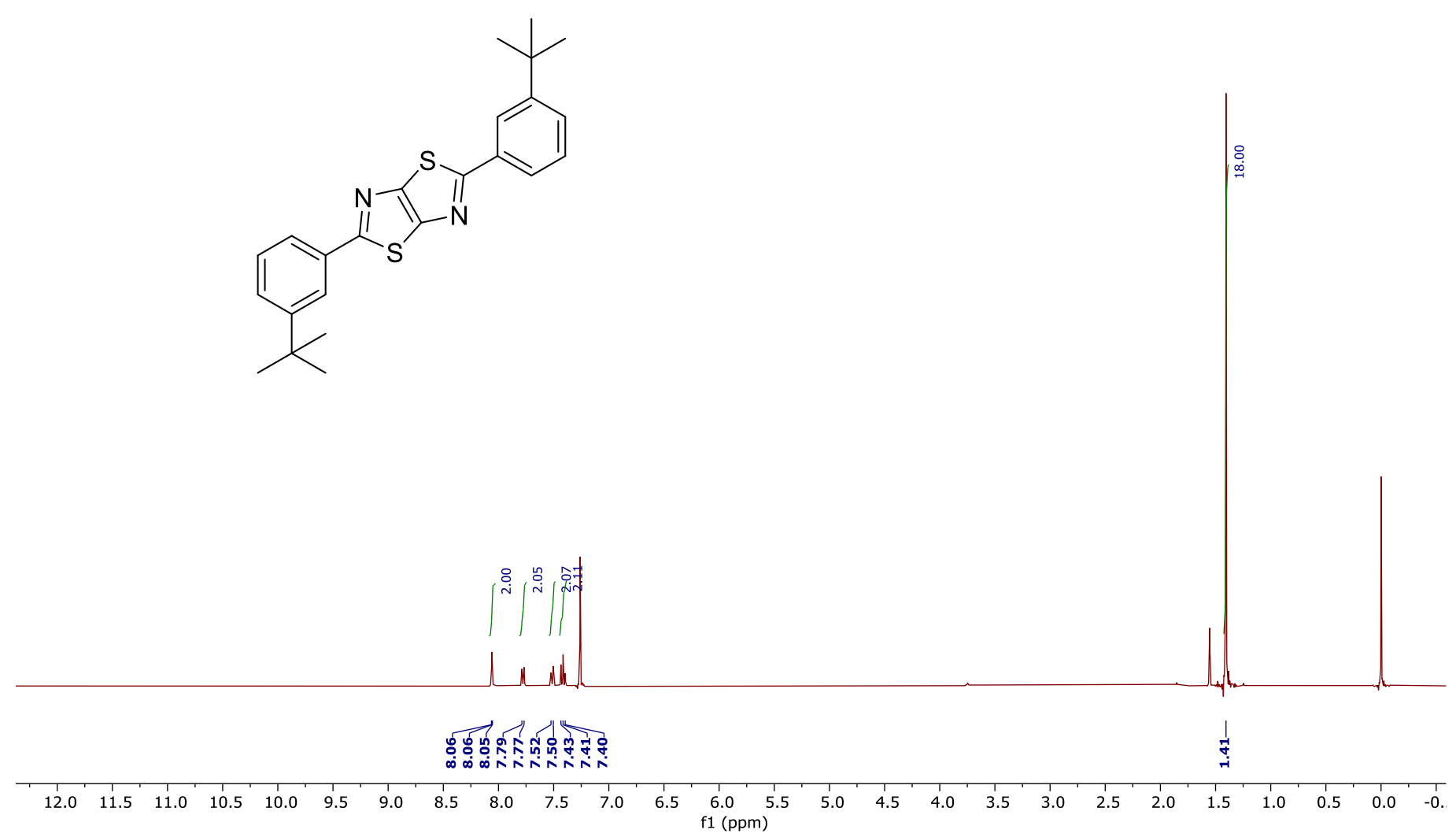

Figure S2. ${ }^{1} \mathrm{H}$ NMR spectrum of proligand 6 in $\mathrm{CDCl}_{3}$ 

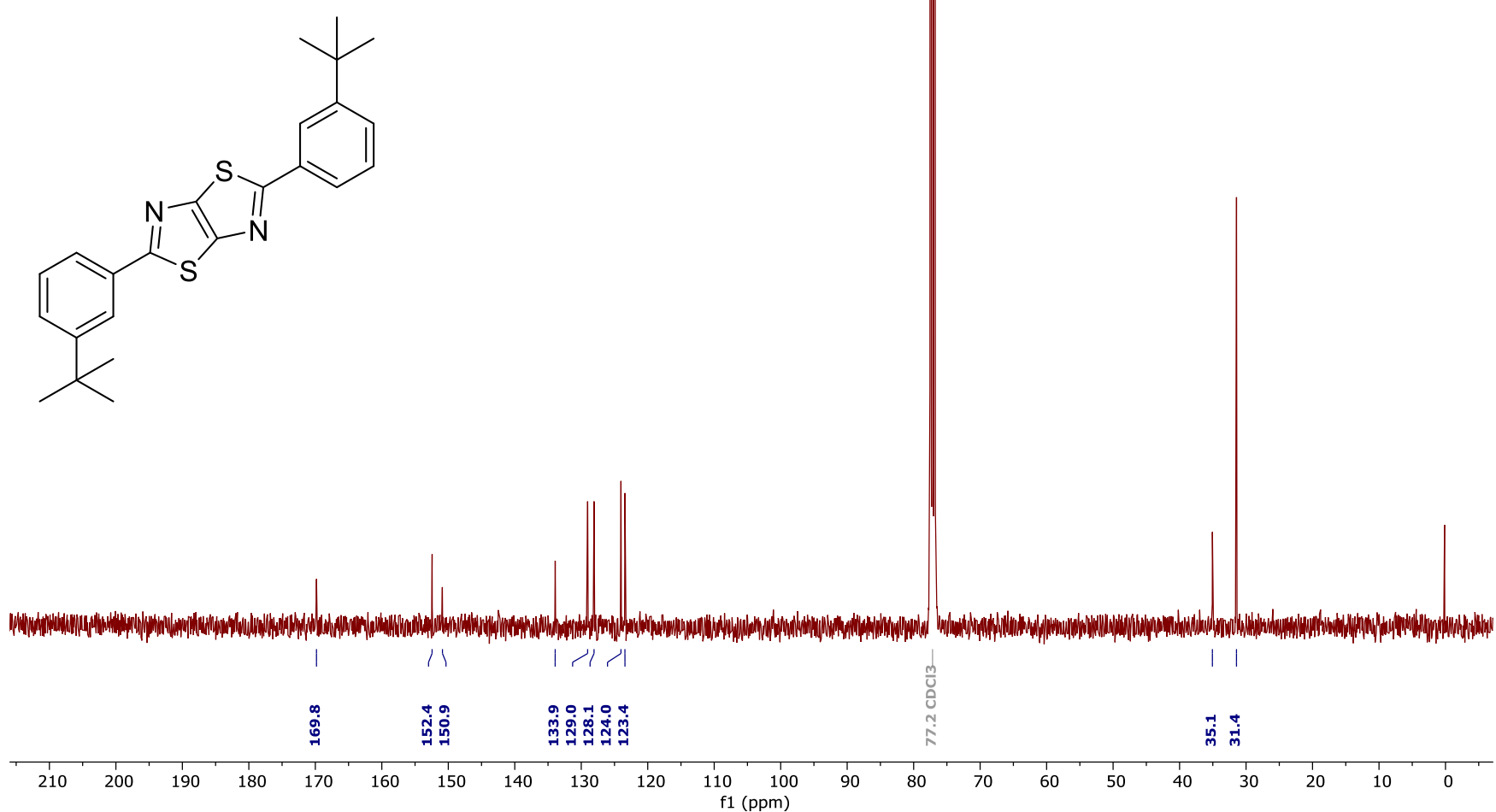

Figure $\mathrm{S3} .{ }^{13} \mathrm{C}$ NMR spectrum of proligand 6 in $\mathrm{CDCl}_{3}$ 
AVZ-VK2-43-1

DEPT with decoupling

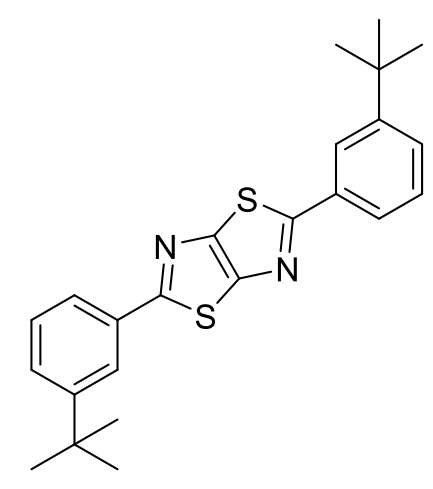

I.w.

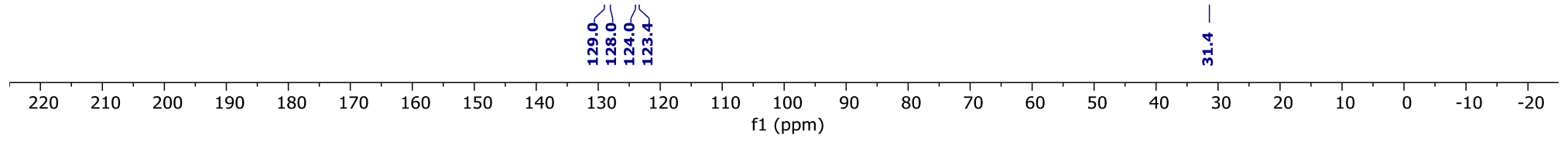

Figure S4. ${ }^{13} \mathrm{C}$ (DEPT135) NMR spectrum of proligand 6 in $\mathrm{CDCl}_{3}$ 
AVZ-VK4-02-3

single_pulse

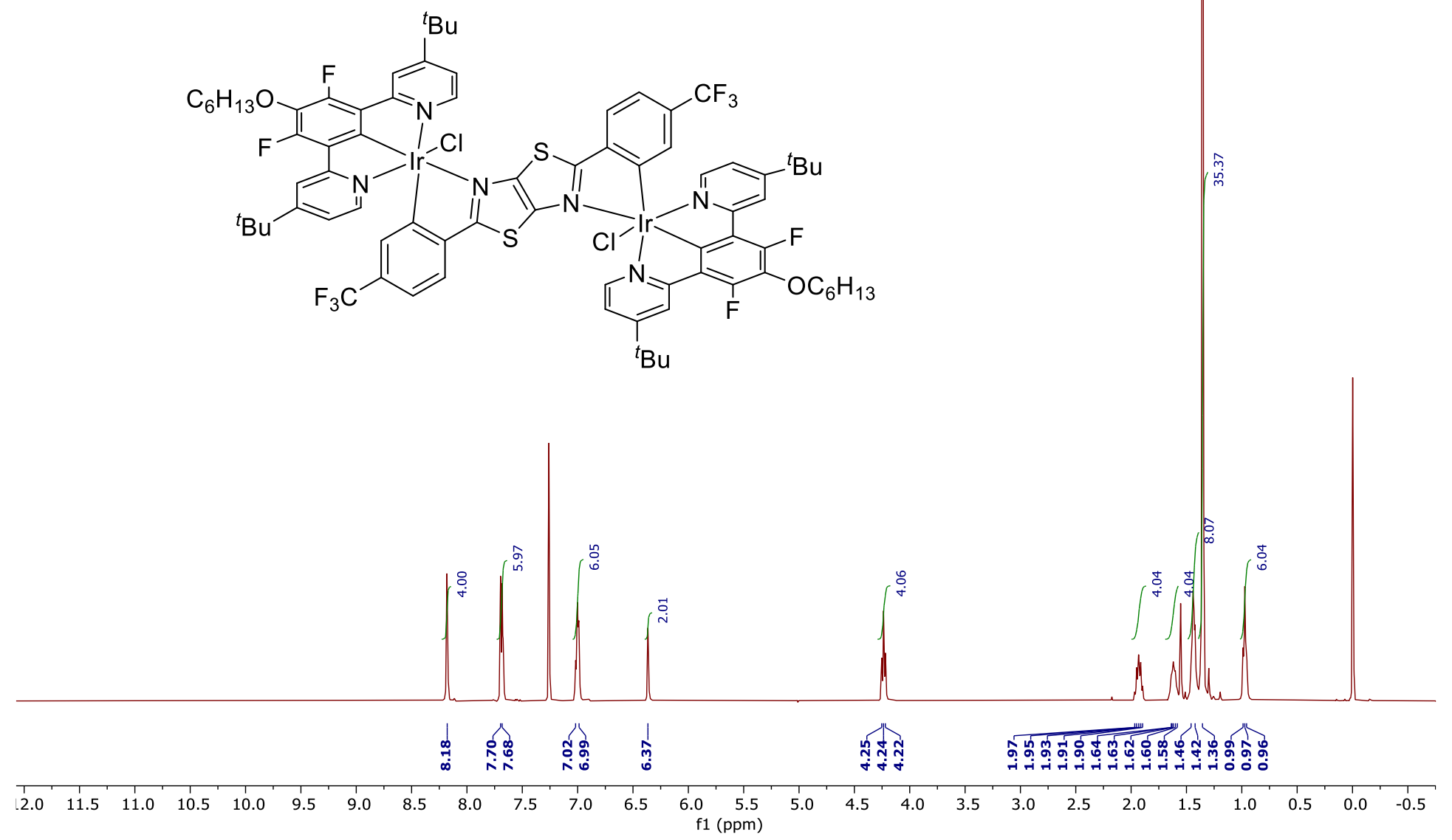

Figure S5. ${ }^{1} \mathrm{H}$ NMR spectrum of complex Ir-1 in $\mathrm{CDCl}_{3}$ 
AVZ-VK4-02-3

single pulse decoupled gated NOE

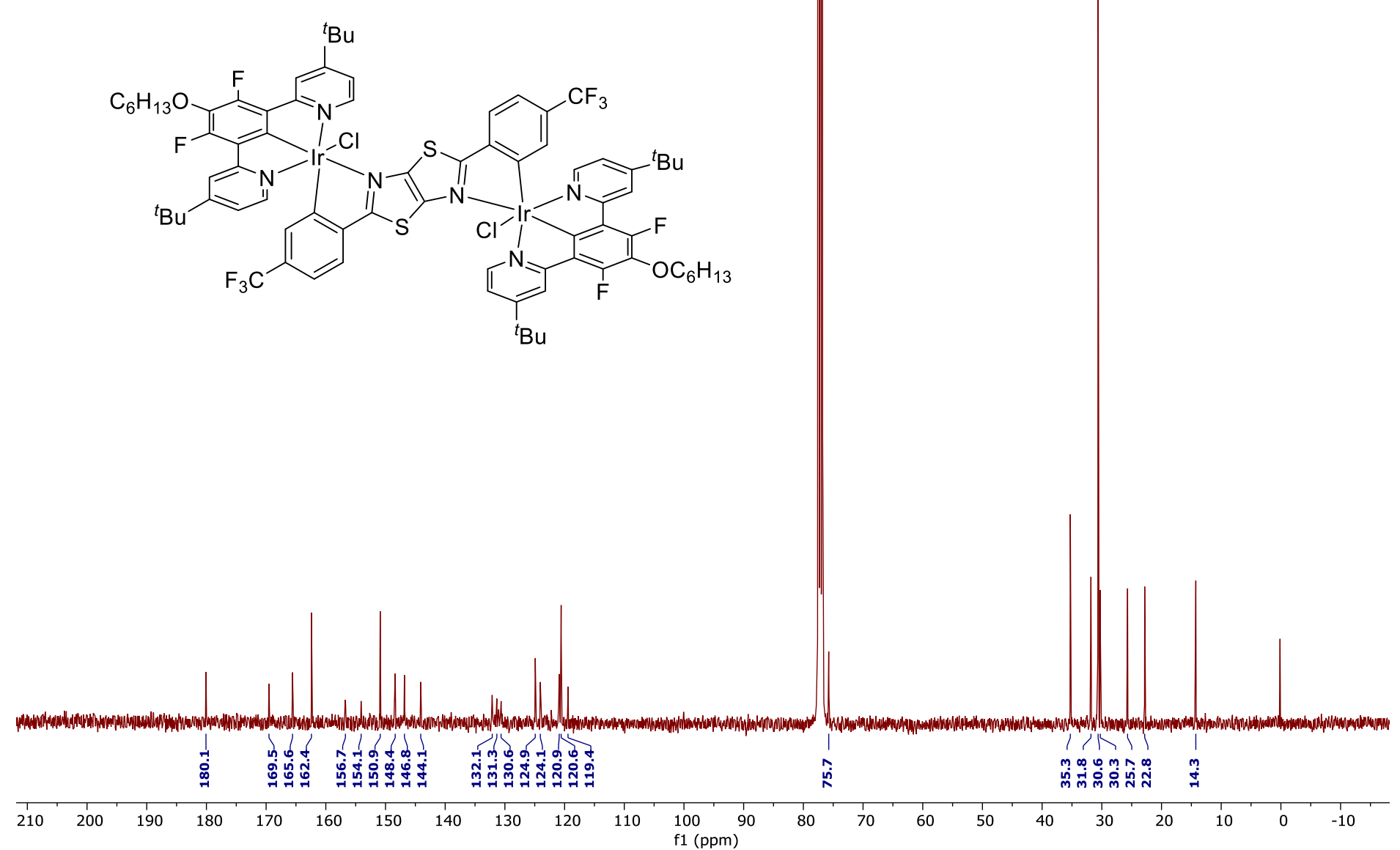

Figure S6. ${ }^{13} \mathrm{C}$ NMR spectrum of complex Ir-1 in $\mathrm{CDCl}_{3}$ 
DEPT with decoupling
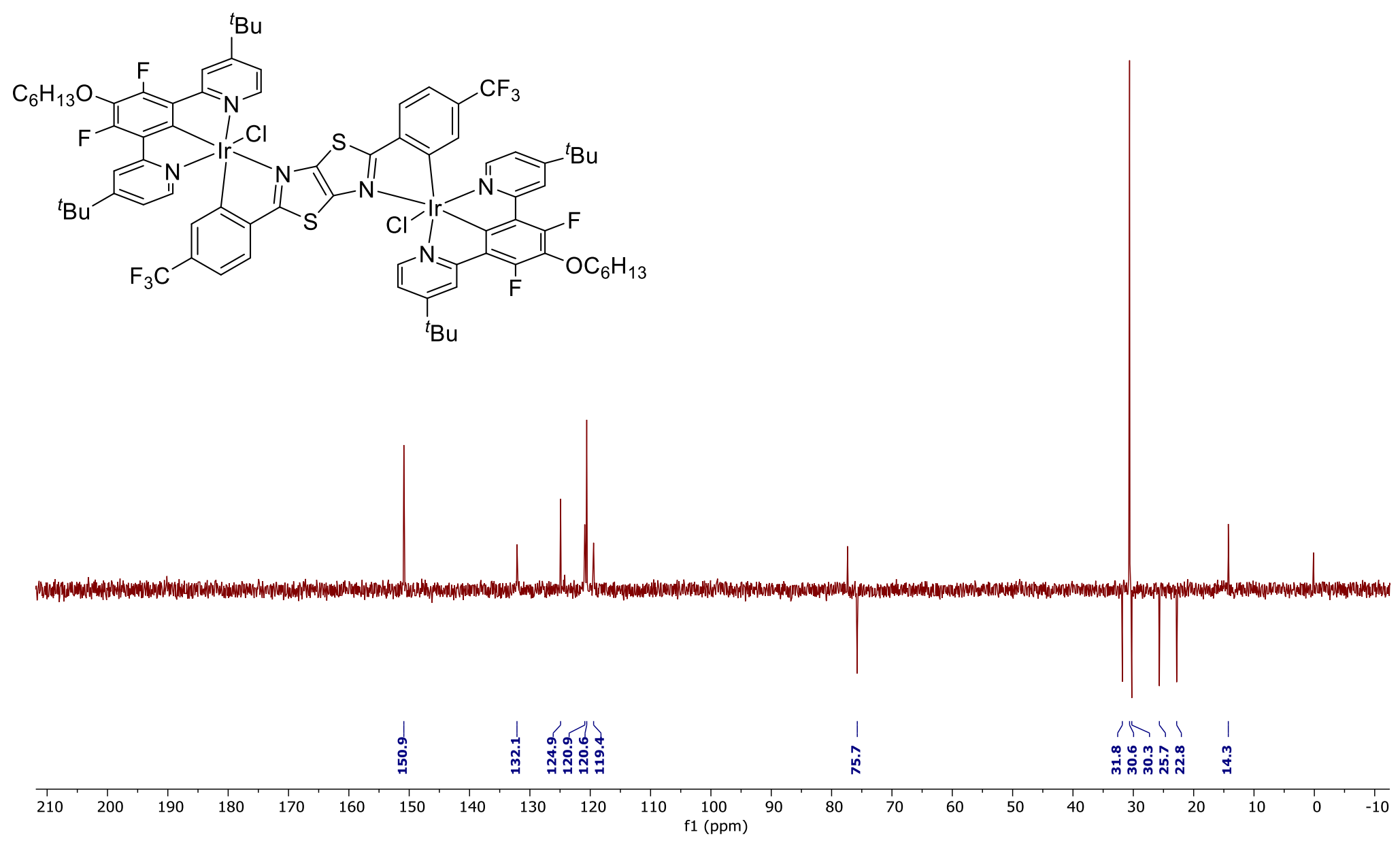

Figure S7. ${ }^{13} \mathrm{C}$ (DEPT135) NMR spectrum of complex Ir-1 in $\mathrm{CDCl}_{3}$ 


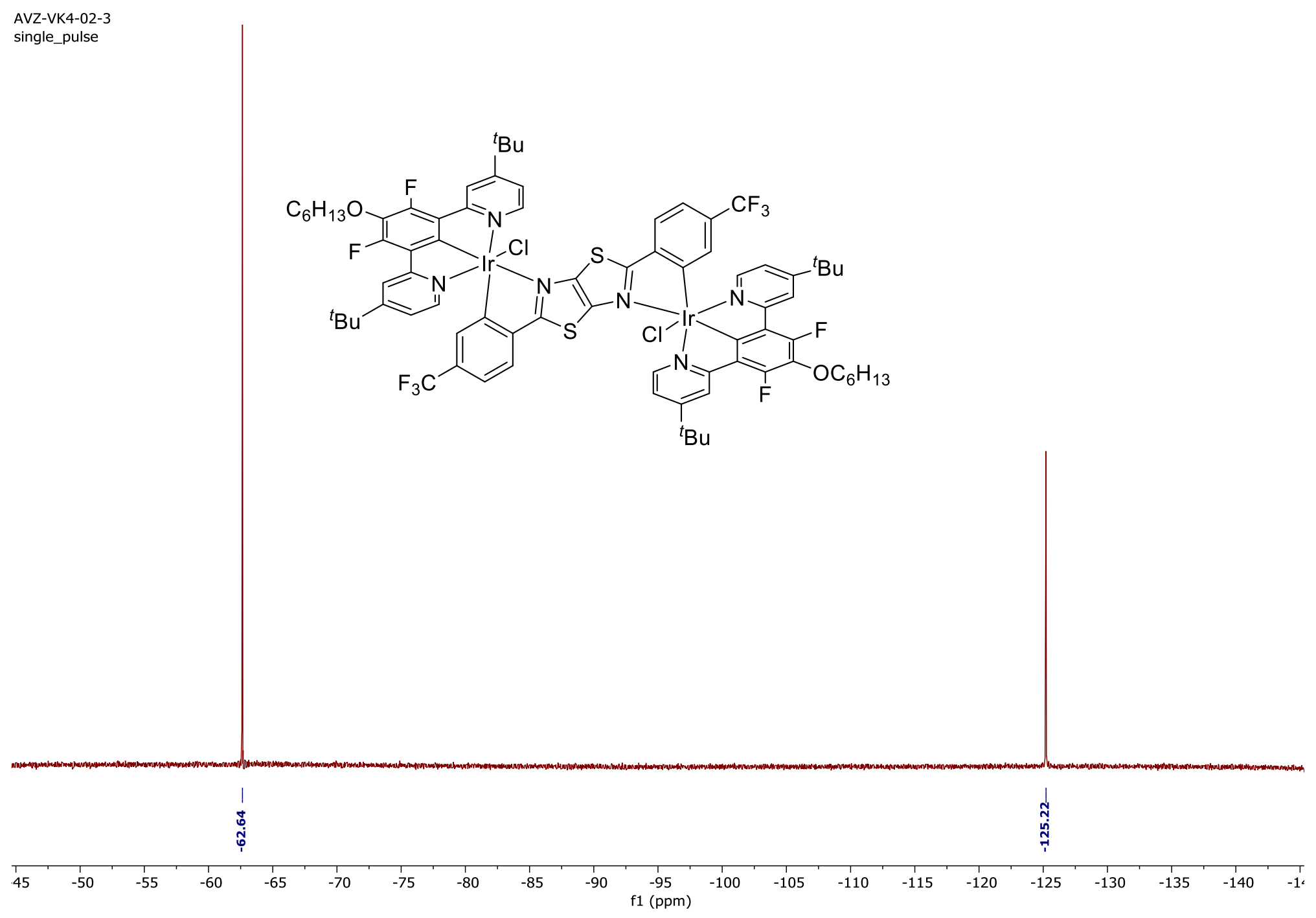

Figure S8. ${ }^{19} \mathrm{~F}$ NMR spectrum of complex Ir-1 in $\mathrm{CDCl}_{3}$ 


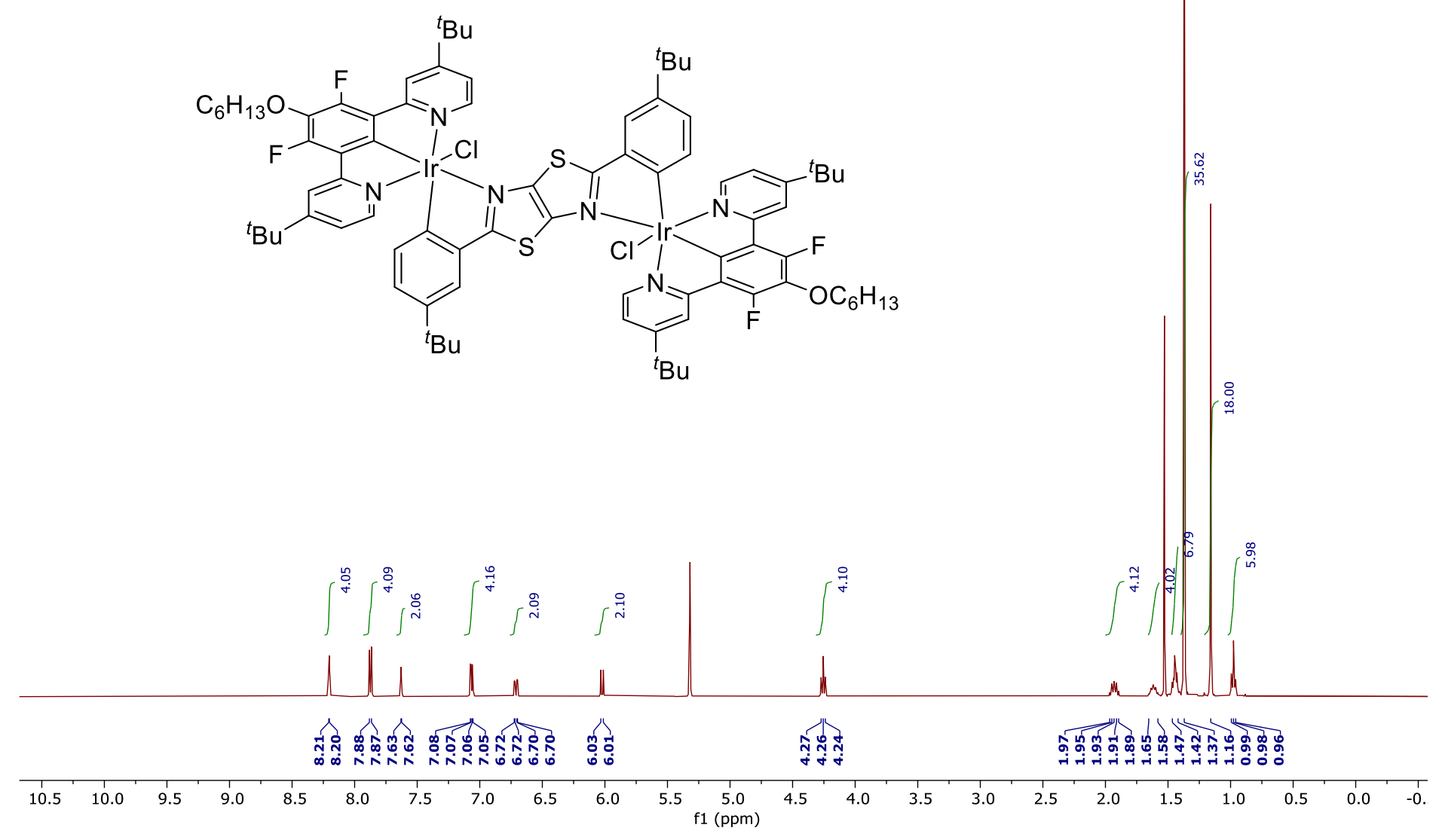

Figure S9. ${ }^{1} \mathrm{H}$ NMR spectrum of complex Ir-2 in $\mathrm{CDCl}_{3}$ 
AVZ-VK2-47-1 2

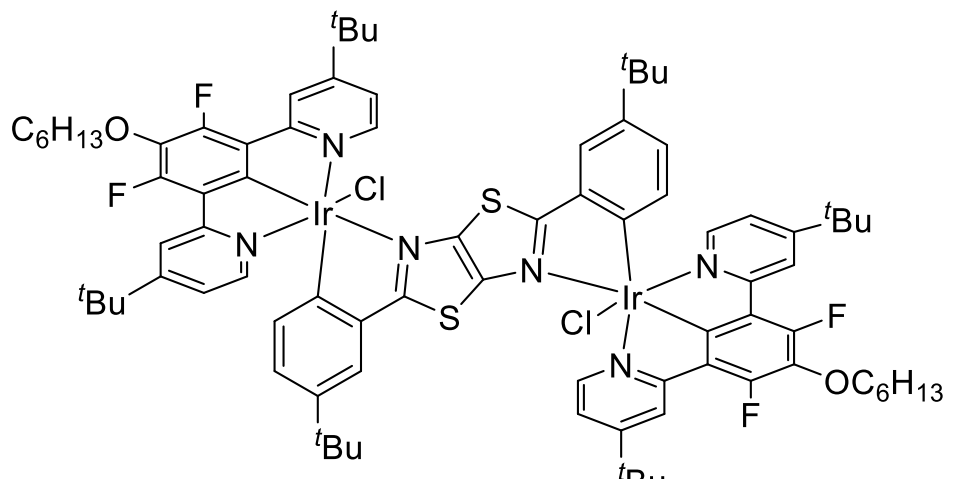

${ }^{t} \mathrm{Bu}$

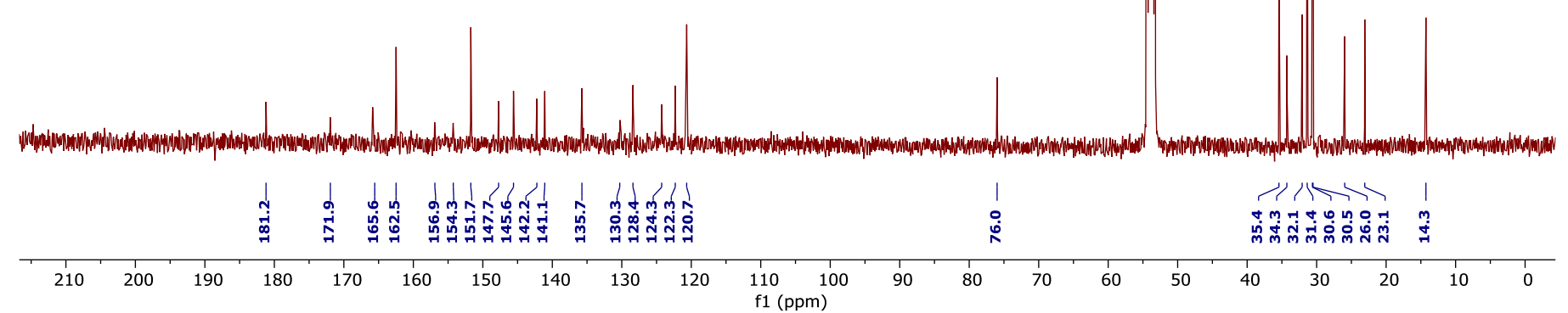

Figure S10. ${ }^{13} \mathrm{C}$ NMR spectrum of complex Ir-2 in $\mathrm{CDCl}_{3}$ 
AVZ-VK2-47-1 2

DEPT with decoupling
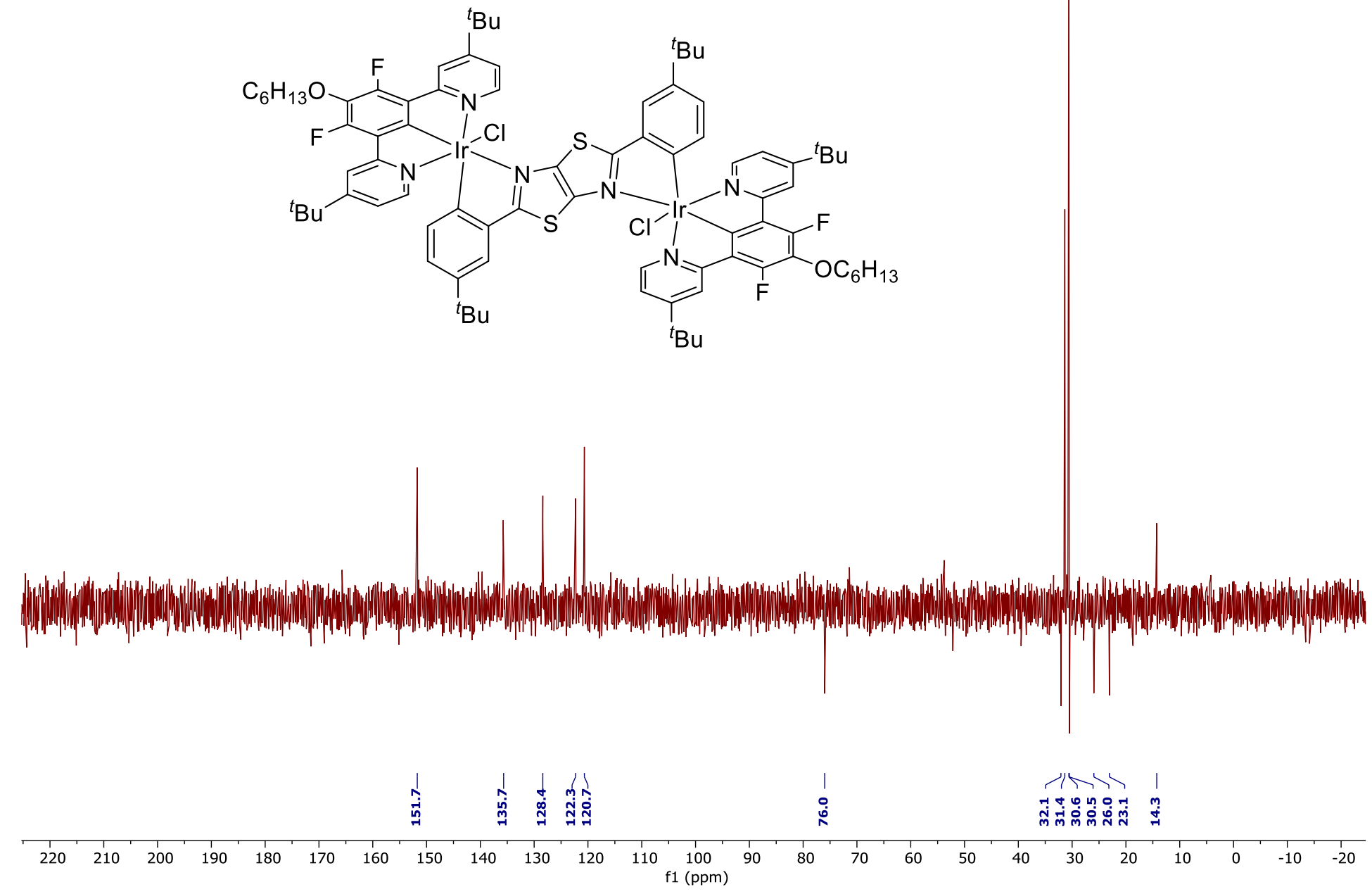

Figure S11. ${ }^{13} \mathrm{C}(\mathrm{DEPT} 135)$ NMR spectrum of complex Ir-2 in $\mathrm{CDCl}_{3}$ 
AVZ-VK2-47-1 1

single_pulse

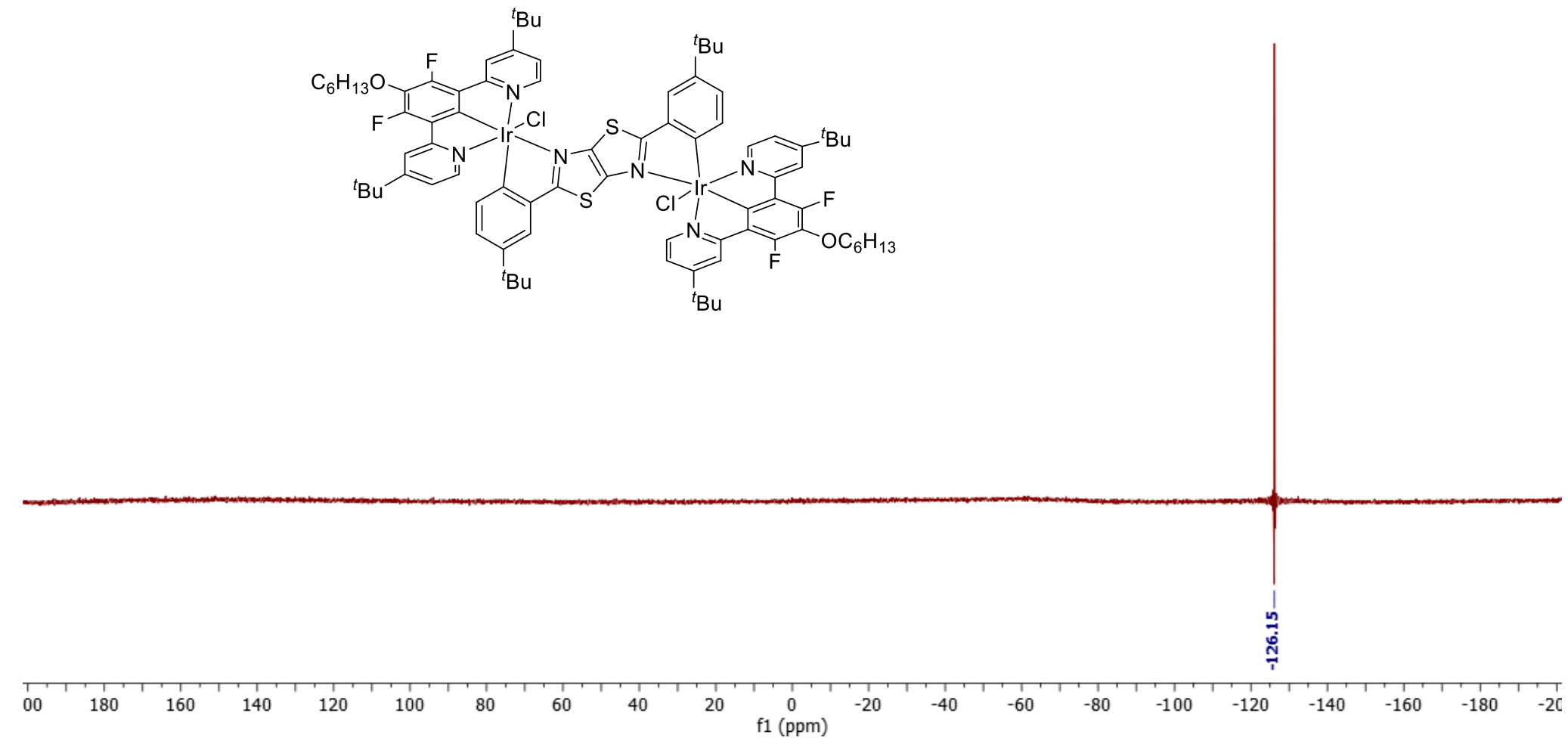

Figure S12. ${ }^{19} \mathrm{~F}$ NMR spectrum of complex Ir-2 in $\mathrm{CDCl}_{3}$ 
Optical spectroscopy. The steady state photophysical measurements were performed on solutions of complexes Ir-1 and Ir-2 in dichloromethane. The UV-Vis absorption spectra were measured with a Varian Cary 300 double beam spectrometer. The emission and excitation spectra were measured with a Horiba Jobin Yvon Fluorolog-3 steady-state fluorescence spectrometer. The emission decay times were measured with a PicoBright PB-375 pulsed diode laser $\left(\lambda_{\mathrm{exc}}=378 \mathrm{~nm}\right.$, pulse width $100 \mathrm{ps}$ ) used as the excitation source, and the PL signal was detected with a cooled photomultiplier attached to a FAST ComTec multichannel scalar PCI card with a time resolution of 250 ps. The PL quantum yield was determined with a Hamamatsu C9920-02 system equipped with a Spectralon ${ }^{\circledR}$ integrating sphere

Computations. All calculations were carried out with the Gaussian 09 package ${ }^{3}$ utilizing the DFT

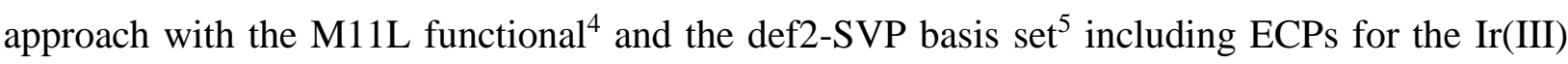
ion. Geometry optimizations were conducted with "tight" criteria. The C-PCM solvation model ${ }^{6}$ applied with solvent parameters for dichloromethane.

Table S1. DFT calculated frontier orbital energies and atomic contributions of complex Ir-1 in the state $\mathrm{T}_{1}$ geometry resulting from Mulliken population analysis.

\begin{tabular}{|c|c|c|c|c|c|c|c|c|}
\hline \multirow[t]{2}{*}{ Orbital } & \multirow{2}{*}{$\begin{array}{l}\text { Energy, } \\
\text { eV }\end{array}$} & \multicolumn{7}{|c|}{ Contribution, \% (Mulliken) } \\
\hline & & Ir1 & Cl1 & $\mathrm{N}^{\wedge} \mathrm{C}^{\wedge} \mathrm{N1}$ & $\mathrm{C}^{\wedge} \mathrm{N}-\mathrm{N}^{\wedge} \mathrm{C}$ & $\mathrm{N}^{\wedge} \mathrm{C}^{\wedge} \mathrm{N2} 2$ & $\mathrm{Cl} 2$ & Ir2 \\
\hline LUMO+4 & -2.655 & 2 & 1 & 41 & 0 & 52 & 1 & 3 \\
\hline LUMO+3 & -2.661 & 2 & 1 & 45 & 15 & 34 & 1 & 2 \\
\hline LUMO+2 & -2.793 & 1 & 0 & 47 & 1 & 50 & 0 & 1 \\
\hline LUMO+1 & -2.817 & 1 & 0 & 44 & 12 & 42 & 0 & 1 \\
\hline LUMO & -3.639 & 1 & 0 & 1 & 97 & 0 & 0 & 1 \\
\hline HOMO & -5.449 & 14 & 5 & 3 & 56 & 3 & 5 & 14 \\
\hline HOMO-1 & -5.639 & 13 & 4 & 15 & 3 & 30 & 9 & 26 \\
\hline HOMO-2 & -5.729 & 25 & 9 & 30 & 3 & 15 & 5 & 13 \\
\hline HOMO-3 & -6.064 & 22 & 12 & 6 & 17 & 7 & 13 & 23 \\
\hline HOMO-4 & -6.257 & 10 & 8 & 7 & 50 & 7 & 8 & 9 \\
\hline
\end{tabular}

$\mathrm{CN}-\mathrm{NC}-$ The $\mathrm{C}^{\wedge} \mathrm{N}-\mathrm{N}^{\wedge} \mathrm{C}$ coordinating ditopic (bis-bidentate) bridging ligand

$\mathrm{NCN} 1-$ The $\mathrm{N}^{\wedge} \mathrm{C}^{\wedge} \mathrm{N}$ coordinating tridentate ligand coordinated to $\mathrm{Ir} 1$

$\mathrm{NCN} 2-$ The $\mathrm{N}^{\wedge} \mathrm{C}^{\wedge} \mathrm{N}$ coordinating tridentate ligand coordinated to $\mathrm{Ir} 2$

Cl1 - The chloride coordinated to Ir1

$\mathrm{Cl} 2$ - The chloride coordinated to Ir2 
Table S2. DFT calculated frontier orbital energies and atomic contributions of complex Ir-2 in the state $\mathrm{T}_{1}$ geometry resulting from Mulliken population analysis.

\begin{tabular}{|c|c|c|c|c|c|c|c|c|}
\hline \multirow[t]{2}{*}{ Orbital } & \multirow{2}{*}{$\begin{array}{l}\text { Energy, } \\
\text { eV }\end{array}$} & \multicolumn{7}{|c|}{ Contribution, \% (Mulliken) } \\
\hline & & Ir1 & $\mathrm{Cl} 1$ & NCN1 & CN-NC & NCN2 & $\mathrm{Cl} 2$ & Ir2 \\
\hline LUMO+4 & -2.593 & 2 & 1 & 47 & 0 & 47 & 1 & 2 \\
\hline LUMO+3 & -2.594 & 2 & 1 & 47 & 0 & 47 & 1 & 2 \\
\hline LUMO+2 & -2.737 & 1 & 0 & 49 & 1 & 48 & 0 & 1 \\
\hline LUMO+1 & -2.748 & 1 & 0 & 48 & 2 & 48 & 0 & 1 \\
\hline LUMO & -3.289 & 1 & 0 & 1 & 96 & 1 & 0 & 1 \\
\hline HOMO & -5.159 & 10 & 3 & 2 & 70 & 2 & 3 & 10 \\
\hline HOMO-1 & -5.530 & 20 & 6 & 22 & 4 & 22 & 6 & 20 \\
\hline HOMO-2 & -5.533 & 19 & 7 & 22 & 4 & 22 & 7 & 19 \\
\hline HOMO-3 & -5.551 & 21 & 10 & 6 & 26 & 6 & 10 & 21 \\
\hline HOMO-4 & -5.837 & 13 & 8 & 6 & 46 & 6 & 8 & 13 \\
\hline
\end{tabular}

$\mathrm{CN}-\mathrm{NC}-$ The $\mathrm{C}^{\wedge} \mathrm{N}-\mathrm{N}^{\wedge} \mathrm{C}$ coordinating ditopic (bis-bidentate) bridging ligand

$\mathrm{NCN} 1$ - The $\mathrm{N}^{\wedge} \mathrm{C}^{\wedge} \mathrm{N}$ coordinating tridentate ligand coordinated to Ir1

$\mathrm{NCN} 2-$ The $\mathrm{N}^{\wedge} \mathrm{C}^{\wedge} \mathrm{N}$ coordinating tridentate ligand coordinated to $\mathrm{Ir} 2$

Cl1 - The chloride coordinated to Ir 1

$\mathrm{Cl} 2$ - The chloride coordinated to Ir2

Table S3. DFT calculated frontier orbital energies and atomic contributions of complex IrIr' in the state $\mathrm{T}_{1}$ geometry resulting from Mulliken population analysis.

\begin{tabular}{|l|l|l|l|l|l|l|l|l|}
\hline \multirow{2}{*}{ Orbitals } & \multirow{2}{\text{Energy,}}{$\mathbf{e V}$} & \multicolumn{7}{|c|}{ Contribution (Mulliken) } \\
\cline { 3 - 9 } & Ir1 & NCN1 & Cl1 & CN-CN & Ir2 & NCN2 & Cl2 \\
\hline LUMO+4 & -2.669 & 2 & 47 & 1 & 0 & 2 & 47 & 1 \\
\hline LUMO+3 & -2.678 & 3 & 46 & 1 & 0 & 3 & 46 & 1 \\
\hline LUMO+2 & -2.810 & 1 & 49 & 0 & 0 & 1 & 49 & 0 \\
\hline LUMO+1 & -2.815 & 1 & 49 & 0 & 0 & 1 & 49 & 0 \\
\hline LUMO & -3.473 & 1 & 1 & 0 & 96 & 1 & 1 & 0 \\
\hline HOMO & -5.168 & 8 & 2 & 2 & 76 & 8 & 2 & 2 \\
\hline HOMO-1 & -5.624 & 20 & 21 & 7 & 4 & 20 & 21 & 7 \\
\hline HOMO-2 & -5.660 & 23 & 7 & 11 & 19 & 23 & 7 & 11 \\
\hline HOMO-3 & -5.665 & 8 & 2 & 7 & 66 & 8 & 2 & 7 \\
\hline HOMO-4 & -5.976 & 19 & 10 & 8 & 26 & 19 & 10 & 8 \\
\hline
\end{tabular}

$\mathrm{NC}-\mathrm{CN}-$ The $\mathrm{N}^{\wedge} \mathrm{C}-\mathrm{C}^{\wedge} \mathrm{N}$ ditopic ligand bridging the two metal centers

$\mathrm{NCN} 1-\mathrm{N}^{\wedge} \mathrm{C}^{\wedge} \mathrm{N}$ ligand coordinated to $\operatorname{Ir} 1$

$\mathrm{NCN} 2-\mathrm{N}^{\wedge} \mathrm{C}^{\wedge} \mathrm{N}$ ligand coordinated to Ir2 
Table S4. TD-DFT calculated lowest triplet and singlet states of Ir-1 in the $\mathrm{T}_{1}$ state geometry

\begin{tabular}{|c|c|c|c|}
\hline $\begin{array}{l}\text { State, } \\
\text { energy } \\
(\mathrm{eV})\end{array}$ & $\begin{array}{c}f \\
\text { (oscillator } \\
\text { strength) }\end{array}$ & $\begin{array}{l}\text { Contributing transition } \\
\text { coefficients* }\end{array}$ & Character** \\
\hline \multicolumn{4}{|c|}{ triplets } \\
\hline $\begin{array}{c}\mathrm{T}_{1} \\
1.612\end{array}$ & (triplet) & $\begin{array}{l}\text { HOMO } \rightarrow \text { LUMO }(0.69) \\
\text { HOMO }-4 \rightarrow \text { LUMO }(-0.16)\end{array}$ & $\mathrm{LC}^{\mathrm{CN}-\mathrm{NC}} / \mathrm{ML}^{\mathrm{CN}-\mathrm{NC}} \mathrm{CT} / \mathrm{XL} \mathrm{L}^{\mathrm{CN}-\mathrm{NC}} \mathrm{CT}$ \\
\hline $\begin{array}{c}\mathrm{T}_{2} \\
1.987\end{array}$ & (triplet) & HOMO-1 $\rightarrow$ LUMO (0.70) & $\mathrm{L}^{\mathrm{NCN}} \mathrm{L}^{\mathrm{CN}-\mathrm{NC}} \mathrm{CT} / \mathrm{ML}^{\mathrm{CN}-\mathrm{NC}} \mathrm{CT} / \mathrm{XL} \mathrm{C}^{\mathrm{CN}-\mathrm{NC}} \mathrm{CT}$ \\
\hline $\begin{array}{c}\mathrm{T}_{3} \\
1.991\end{array}$ & (triplet) & HOMO-2 $\rightarrow$ LUMO (0.70) & $\mathrm{L}^{\mathrm{NCN}} \mathrm{L}^{\mathrm{CN}-\mathrm{NC}} \mathrm{CT} / \mathrm{ML}^{\mathrm{CN}-\mathrm{NC}} \mathrm{CT} / \mathrm{XL} \mathrm{C}^{\mathrm{CN}-\mathrm{NC}} \mathrm{CT}$ \\
\hline $\begin{array}{c}\mathrm{T}_{4} \\
2.058\end{array}$ & (triplet) & HOMO-3 $\rightarrow$ LUMO (0.70) & $\mathrm{ML}^{\mathrm{CN}-\mathrm{NC}} \mathrm{CT} / \mathrm{XL}^{\mathrm{CN}-\mathrm{NC}} \mathrm{CT}$ \\
\hline $\begin{array}{c}T_{5} \\
2.273\end{array}$ & (triplet) & $\begin{array}{l}\text { HOMO-4 } \rightarrow \text { LUMO }(0.68) \\
\text { HOMO } \rightarrow \text { LUMO }(0.16)\end{array}$ & $\mathrm{LC}^{\mathrm{CN}-\mathrm{NC}} / \mathrm{ML}^{\mathrm{CN}-\mathrm{NC}} \mathrm{CT} / \mathrm{XL} \mathrm{C}^{\mathrm{CN}-\mathrm{NC}} \mathrm{CT} / \mathrm{L}^{\mathrm{NCN}} \mathrm{L}^{\mathrm{CN}-\mathrm{NC}} \mathrm{CT}$ \\
\hline \multicolumn{4}{|c|}{ singlets } \\
\hline $\begin{array}{c}\mathrm{S}_{1} \\
1.983\end{array}$ & 0.3084 & $\begin{array}{l}\text { HOMO } \rightarrow \text { LUMO (0.65) } \\
\text { HOMO-4 } \rightarrow \text { LUMO (0.19) } \\
\text { HOMO-2 } \rightarrow \text { LUMO }(-0.16)\end{array}$ & $\mathrm{LC}^{\mathrm{CN}-\mathrm{NC}} / \mathrm{ML}^{\mathrm{CN}-\mathrm{NC}} \mathrm{CT} / \mathrm{XL}^{\mathrm{CN}-\mathrm{NC}} \mathrm{CT}$ \\
\hline $\begin{array}{l}\mathrm{S}_{2} \\
1.996\end{array}$ & 0.0017 & HOMO-1 $\rightarrow$ LUMO (0.70) & $\mathrm{L}^{\mathrm{CNC}} \mathrm{L}^{\mathrm{CN}-\mathrm{NC}} \mathrm{CT} / \mathrm{ML}^{\mathrm{CN}-\mathrm{NC}} \mathrm{CT} / \mathrm{XL}^{\mathrm{CN}-\mathrm{NC}} \mathrm{CT}$ \\
\hline $\begin{array}{c}\mathrm{S}_{3} \\
2.002\end{array}$ & 0.0233 & $\begin{array}{l}\text { HOMO-2 } \rightarrow \text { LUMO (0.68) } \\
\text { HOMO } \rightarrow \text { LUMO }(0.16)\end{array}$ & $\mathrm{L}^{\mathrm{NCN}} \mathrm{L}^{\mathrm{CN}-\mathrm{NC}} \mathrm{CT} / \mathrm{ML}^{\mathrm{CN}-\mathrm{NC}} \mathrm{CT} / \mathrm{XL}{ }^{\mathrm{CN}-\mathrm{NC}} \mathrm{CT}$ \\
\hline $\begin{array}{l}\mathrm{S}_{4} \\
2.129\end{array}$ & 0.0000 & HOMO-3 $\rightarrow$ LUMO (0.70) & $\mathrm{ML}^{\mathrm{CN}-\mathrm{NC}} \mathrm{CT} / \mathrm{XL}{ }^{\mathrm{CN}-\mathrm{NC}} \mathrm{CT}$ \\
\hline $\begin{array}{c}\mathrm{S}_{5} \\
2.651\end{array}$ & 0.2011 & $\begin{array}{l}\text { HOMO }-4 \rightarrow \text { LUMO }(0.45) \\
\text { HOMO } \rightarrow \text { LUMO+2 }(0.42) \\
\text { HOMO-5 } \rightarrow \text { LUMO }(-0.24) \\
\text { HOMO-9 } \rightarrow \text { LUMO }(0.15) \\
\text { HOMO } \rightarrow \text { LUMO }(-0.10) \\
\text { HOMO } \rightarrow \text { LUMO+1 }(-0.11)\end{array}$ & $\begin{array}{c}\mathrm{L}^{\mathrm{CN}-\mathrm{NC} C} \mathrm{C} / \mathrm{L}^{\mathrm{CN}-\mathrm{NC}} \mathrm{L}^{\mathrm{NCN}} \mathrm{CT} / \mathrm{ML}^{\mathrm{CN}-\mathrm{NC}} \mathrm{CT} / \mathrm{ML}^{\mathrm{NCN}} \mathrm{CT} \\
/ \mathrm{XL}^{\mathrm{CN}-\mathrm{NC}} \mathrm{CT} / \mathrm{XL}^{\mathrm{NCN}} \mathrm{CT}\end{array}$ \\
\hline
\end{tabular}

* Square of the coefficient multiplied by two gives percentage contribution of the transition to formation of the excited state.

**MLCT - Metal (M) to Ligand (L) Charge Transfer. XLCT - Halide (X) to Ligand (L) Charge Transfer. LC-Ligand Centered. LLCT - Ligand to Ligand Charge Transfer. 
Table S5. TD-DFT calculated lowest triplet and singlet states of Ir-2 in the $\mathrm{T}_{1}$ state geometry

\begin{tabular}{|c|c|c|c|}
\hline $\begin{array}{l}\text { State, } \\
\text { energy } \\
(\mathrm{eV})\end{array}$ & $\begin{array}{c}f \\
\text { (oscillator } \\
\text { strength) }\end{array}$ & $\begin{array}{l}\text { Contributing transition } \\
\text { coefficients* }\end{array}$ & Character** \\
\hline \multicolumn{4}{|c|}{ triplets } \\
\hline $\begin{array}{c}\mathrm{T}_{1} \\
1.639\end{array}$ & (triplet) & $\begin{array}{l}\text { HOMO } \rightarrow \text { LUMO (0.69) } \\
\text { HOMO-4 } \rightarrow \text { LUMO (0.13) }\end{array}$ & $\mathrm{LC}^{\mathrm{CN}-\mathrm{NC}} / \mathrm{ML}^{\mathrm{CN}-\mathrm{NC}} \mathrm{CT} / \mathrm{XL}{ }^{\mathrm{CN}-\mathrm{NC}} \mathrm{CT}$ \\
\hline $\begin{array}{c}\mathrm{T}_{2} \\
2.217\end{array}$ & (triplet) & $\begin{array}{l}\text { HOMO-3 } \rightarrow \text { LUMO }(0.66) \\
\text { HOMO-1 } \rightarrow \text { LUMO }(-0.25)\end{array}$ & $\mathrm{ML}^{\mathrm{CN}-\mathrm{NC}} \mathrm{CT} / \mathrm{LC} C^{\mathrm{NC}-\mathrm{CN}} / \mathrm{XL}^{\mathrm{CN}-\mathrm{NC}} \mathrm{CT} / \mathrm{L}^{\mathrm{CNC}} \mathrm{L}^{\mathrm{CN}-\mathrm{NC}} \mathrm{CT}$ \\
\hline $\begin{array}{c}\mathrm{T}_{3} \\
2.234\end{array}$ & (triplet) & $\begin{array}{l}\text { HOMO-1 } \rightarrow \text { LUMO }(0.66) \\
\text { HOMO-3 } \rightarrow \text { LUMO }(0.25)\end{array}$ & $\mathrm{L}^{\mathrm{NCN}} \mathrm{L}^{\mathrm{CN}-\mathrm{NC}} \mathrm{CT} / \mathrm{ML}^{\mathrm{CN}-\mathrm{NC}} \mathrm{CT} / \mathrm{X} \mathrm{L}^{\mathrm{CN}-\mathrm{NC}} \mathrm{CT}$ \\
\hline $\begin{array}{c}\mathrm{T}_{4} \\
2.235 \\
\end{array}$ & (triplet) & HOMO-2 $\rightarrow$ LUMO (0.70) & $\mathrm{L}^{\mathrm{NCN}} \mathrm{L}^{\mathrm{CN}-\mathrm{NC}} \mathrm{CT} / \mathrm{ML}^{\mathrm{CN}-\mathrm{NC}} \mathrm{CT} / \mathrm{XL}^{\mathrm{CN}-\mathrm{NC}} \mathrm{CT}$ \\
\hline $\begin{array}{c}\mathrm{T}_{5} \\
2.390\end{array}$ & (triplet) & HOMO $\rightarrow$ LUMO+1 (0.70) & 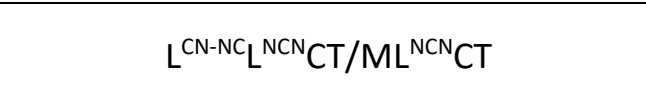 \\
\hline \multicolumn{4}{|c|}{ singlets } \\
\hline $\begin{array}{c}\mathrm{S}_{1} \\
2.104\end{array}$ & 0.5123 & $\begin{array}{l}\text { HOMO } \rightarrow \text { LUMO }(0.67) \\
\text { HOMO-4 } \rightarrow \text { LUMO }(-0.20)\end{array}$ & $\mathrm{LC}^{\mathrm{CN}-\mathrm{NC}} / \mathrm{ML}^{\mathrm{CN}-\mathrm{NC}} \mathrm{CT} / \mathrm{XL}^{\mathrm{CN}-\mathrm{NC}} \mathrm{CT}$ \\
\hline $\begin{array}{c}\mathrm{S}_{2} \\
2.243\end{array}$ & 0.0000 & HOMO-1 $\rightarrow$ LUMO (0.70) & $\mathrm{L}^{\mathrm{NCN}} \mathrm{L}^{\mathrm{CN}-\mathrm{NC}} \mathrm{CT} / \mathrm{ML}^{\mathrm{CN}-\mathrm{NC}} \mathrm{CT} / \mathrm{XL}^{\mathrm{CN}-\mathrm{NC}} \mathrm{CT}$ \\
\hline $\begin{array}{c}\mathrm{S}_{3} \\
2.245\end{array}$ & 0.0014 & HOMO-2 $\rightarrow$ LUMO (0.70) & $\mathrm{L}^{\mathrm{NCN}} \mathrm{L}^{\mathrm{CN}-\mathrm{NC}} \mathrm{CT} / \mathrm{ML}^{\mathrm{CN}-\mathrm{NC}} \mathrm{CT} / \mathrm{XL}{ }^{\mathrm{CN}-\mathrm{NC}} \mathrm{CT}$ \\
\hline $\begin{array}{c}\mathrm{S}_{4} \\
2.315\end{array}$ & 0.0000 & HOMO-3 $\rightarrow$ LUMO (0.69) & $M L^{C N-N C} C T / L C^{N C-C N} / X^{C N-N C} C T / L^{C N C} L^{C N-N C} C T$ \\
\hline $\begin{array}{c}\mathrm{S}_{5} \\
2.422\end{array}$ & 0.0000 & HOMO $\rightarrow$ LUMO+1 (0.70) & 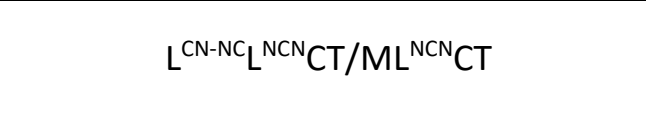 \\
\hline
\end{tabular}

* Square of the coefficient multiplied by two gives percentage contribution of the transition to formation of the excited state.

**MLCT - Metal (M) to Ligand (L) Charge Transfer. XLCT - Halide (X) to Ligand (L) Charge Transfer. LC-Ligand Centered. LLCT - Ligand to Ligand Charge Transfer. 
Table S6. TD-DFT calculated lowest triplet and singlet states of IrIr' in the $\mathrm{T}_{1}$ state geometry.

\begin{tabular}{|c|c|c|c|}
\hline $\begin{array}{l}\text { State, } \\
\text { energy } \\
(\mathrm{eV})\end{array}$ & $\begin{array}{c}f \\
\text { (oscillator } \\
\text { strength) }\end{array}$ & $\begin{array}{l}\text { Contributing transition } \\
\text { coefficients* }\end{array}$ & Character** \\
\hline \multicolumn{4}{|c|}{ triplets } \\
\hline $\begin{array}{c}\mathrm{T}_{1} \\
1.514\end{array}$ & (triplet) & HOMO $\rightarrow$ LUMO $(0.70)$ & 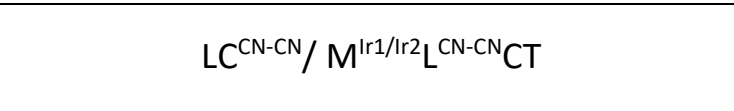 \\
\hline $\begin{array}{c}\mathrm{T}_{2} \\
2.124 \\
\end{array}$ & (triplet) & HOMO-2 $\rightarrow$ LUMO (0.70) & 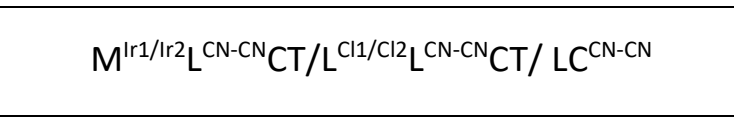 \\
\hline $\begin{array}{c}\mathrm{T}_{3} \\
2.140\end{array}$ & (triplet) & HOMO-1 $\rightarrow$ LUMO (0.70) & 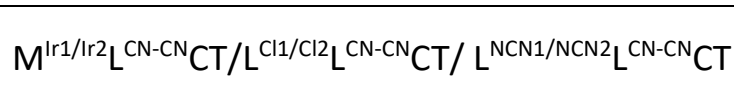 \\
\hline $\begin{array}{c}\mathrm{T}_{4} \\
2.182\end{array}$ & (triplet) & HOMO-3 $\rightarrow$ LUMO (0.70) & $\mathrm{LC}^{\mathrm{CN}-\mathrm{CN}} / \mathrm{M}^{\mathrm{Ir} 1 / \mathrm{Ir} 2} \mathrm{~L}^{\mathrm{CN}-\mathrm{CN}} \mathrm{CT} / \mathrm{L}^{\mathrm{Cl} 1 / \mathrm{Cl} 2} \mathrm{~L}^{\mathrm{CN}-\mathrm{CN}} \mathrm{CT}$ \\
\hline $\begin{array}{c}\mathrm{T}_{5} \\
2.344\end{array}$ & (triplet) & HOMO $\rightarrow$ LUMO+1 (0.70) & 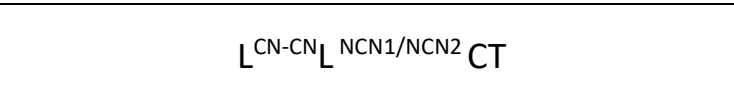 \\
\hline \multicolumn{4}{|c|}{ singlets } \\
\hline $\begin{array}{c}\mathrm{S}_{1} \\
2.048\end{array}$ & 0.2915 & $\begin{array}{l}\text { HOMO } \rightarrow \text { LUMO }(0.68) \\
\text { HOMO-4 } \rightarrow \text { LUMO }(-0.15)\end{array}$ & $\mathrm{LC}^{\mathrm{CN}-\mathrm{CN}} / \mathrm{M}^{\mathrm{Ir} 1 / \mathrm{r} 2 \mathrm{~L}} \mathrm{~L}^{\mathrm{CN}-\mathrm{CN}} \mathrm{CT}$ \\
\hline $\begin{array}{l}\mathrm{S}_{2,} \\
2.153\end{array}$ & 0.0000 & HOMO-1 $\rightarrow$ LUMO (0.70) & 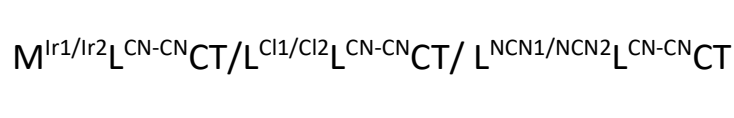 \\
\hline $\begin{array}{l}\mathrm{S}_{3} \\
2.194\end{array}$ & 0.0012 & HOMO-3 $\rightarrow$ LUMO (0.71) & 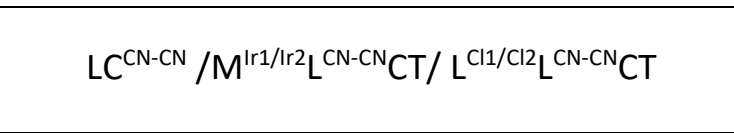 \\
\hline $\begin{array}{c}\mathrm{S}_{4} \\
2.252\end{array}$ & 0.0000 & HOMO-2 $\rightarrow$ LUMO $(0.70)$ & 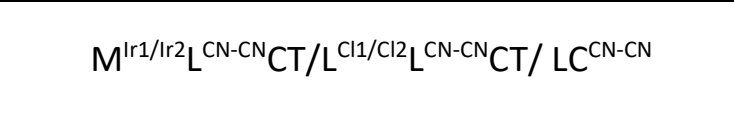 \\
\hline $\begin{array}{l}\mathrm{S}_{5} \\
2.359\end{array}$ & 0.0184 & HOMO $\rightarrow$ LUMO+1 (0.71) & 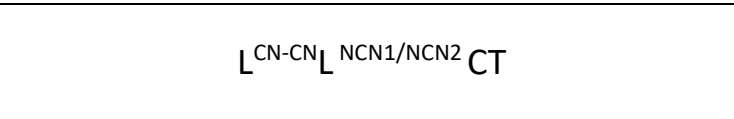 \\
\hline
\end{tabular}

*Square of the coefficient multiplied by two gives percentage contribution of the transition to formation of the excited state.

**MLCT - Metal (M) to Ligand (L) Charge Transfer. LC-Ligand Centered. LLCT - Ligand to Ligand Charge Transfer. 
Table S7. DFT optimized ground state $\left(\mathrm{S}_{0}\right)$ and $\mathrm{T}_{1}$ state geometry of Ir-1' in cartesian (XYZ) coordinates

\begin{tabular}{|c|c|c|c|c|c|c|c|}
\hline \multicolumn{4}{|c|}{ State $S_{0}$} & \multicolumn{4}{|c|}{ State $T_{1}$} \\
\hline $\mathrm{N}$ & 1.743696000 & 0.008217000 & -0.143126000 & $\mathrm{~N}$ & 1.735510000 & 0.007618000 & -0.112230000 \\
\hline C & 0.538074000 & 0.007060000 & 0.429984000 & C & 0.555227000 & 0.006447000 & 0.443444000 \\
\hline C & -0.541170000 & -0.001711000 & -0.433780000 & $\mathrm{C}$ & -0.556525000 & -0.005982000 & -0.443866000 \\
\hline$S$ & 0.002499000 & -0.009020000 & -2.059095000 & $S$ & -0.016146000 & -0.017839000 & -2.076365000 \\
\hline C & 1.626386000 & 0.000069000 & -1.457152000 & $\mathrm{C}$ & 1.632162000 & -0.004510000 & -1.464392000 \\
\hline$S$ & -0.005817000 & 0.012951000 & 2.055415000 & $S$ & 0.014889000 & 0.018099000 & 2.075896000 \\
\hline C & -1.629660000 & 0.002483000 & 1.453277000 & $\mathrm{C}$ & -1.633499000 & 0.004861000 & 1.463978000 \\
\hline $\mathrm{N}$ & -1.746837000 & -0.004123000 & 0.139119000 & $\mathrm{~N}$ & -1.736770000 & -0.006941000 & 0.111779000 \\
\hline C & -2.828314000 & 0.000006000 & 2.249426000 & $\mathrm{C}$ & -2.802239000 & 0.005623000 & 2.236911000 \\
\hline C & 2.824814000 & -0.002041000 & -2.254089000 & $\mathrm{C}$ & 2.800718000 & -0.005171000 & -2.237880000 \\
\hline C & 4.034689000 & 0.005341000 & -1.510949000 & $\mathrm{C}$ & 4.035184000 & 0.007486000 & -1.499219000 \\
\hline C & 5.216231000 & 0.003812000 & -2.248600000 & $\mathrm{C}$ & 5.214431000 & 0.012875000 & -2.233316000 \\
\hline C & 5.204470000 & -0.005084000 & -3.639548000 & $\mathrm{C}$ & 5.204618000 & 0.006368000 & -3.626195000 \\
\hline C & 4.007817000 & -0.012352000 & -4.347581000 & $\mathrm{C}$ & 3.995085000 & -0.008935000 & -4.332911000 \\
\hline C & 2.816906000 & -0.009679000 & -3.649658000 & $\mathrm{C}$ & 2.805518000 & -0.013334000 & -3.650974000 \\
\hline C & -2.821059000 & 0.001620000 & 3.646825000 & $\mathrm{C}$ & -2.807455000 & 0.013597000 & 3.650481000 \\
\hline C & -4.011228000 & -0.003916000 & 4.341271000 & $\mathrm{C}$ & -3.996825000 & 0.010506000 & 4.330962000 \\
\hline C & -5.210678000 & -0.011323000 & 3.631757000 & $\mathrm{C}$ & -5.207486000 & -0.001241000 & 3.623506000 \\
\hline C & -5.221909000 & -0.012636000 & 2.243642000 & $\mathrm{C}$ & -5.217124000 & -0.008100000 & 2.231927000 \\
\hline C & -4.036474000 & -0.007810000 & 1.507255000 & $\mathrm{C}$ & -4.036423000 & -0.005157000 & 1.498328000 \\
\hline Ir & -3.832460000 & -0.012178000 & -0.490968000 & Ir & -3.825466000 & -0.014060000 & -0.488122000 \\
\hline $\mathrm{Cl}$ & -3.200425000 & -0.015261000 & -2.895852000 & $\mathrm{Cl}$ & -3.221804000 & -0.024174000 & -2.893038000 \\
\hline Ir & 3.829434000 & 0.016519000 & 0.487406000 & Ir & 3.824241000 & 0.016035000 & 0.487607000 \\
\hline $\mathrm{Cl}$ & 3.196637000 & 0.027490000 & 2.891985000 & $\mathrm{Cl}$ & 3.220374000 & 0.025637000 & 2.892784000 \\
\hline C & 3.557374000 & 4.335112000 & 0.398797000 & $\mathrm{C}$ & 3.556265000 & 4.333274000 & 0.392865000 \\
\hline C & 3.257865000 & 2.992632000 & 0.344706000 & $\mathrm{C}$ & 3.256111000 & 2.991263000 & 0.336810000 \\
\hline C & 4.864742000 & 4.743847000 & 0.670801000 & $\mathrm{C}$ & 4.862515000 & 4.741062000 & 0.672222000 \\
\hline C & 5.800777000 & 3.738719000 & 0.869947000 & $\mathrm{C}$ & 5.797756000 & 3.735912000 & 0.875799000 \\
\hline C & 5.442686000 & 2.394170000 & 0.802204000 & $\mathrm{C}$ & 5.439864000 & 2.391774000 & 0.805118000 \\
\hline $\mathrm{N}$ & 4.158614000 & 2.039888000 & 0.542703000 & $\mathrm{~N}$ & 4.156636000 & 2.039313000 & 0.539611000 \\
\hline C & 6.340705000 & 1.263068000 & 0.988849000 & $\mathrm{C}$ & 6.336809000 & 1.259867000 & 0.993608000 \\
\hline C & 5.707144000 & 0.022601000 & 0.867704000 & $\mathrm{C}$ & 5.704909000 & 0.020212000 & 0.868841000 \\
\hline C & 6.346417000 & -1.213279000 & 1.000532000 & $\mathrm{C}$ & 6.340789000 & -1.216182000 & 1.001081000 \\
\hline C & 7.705684000 & 1.234492000 & 1.254682000 & $\mathrm{C}$ & 7.701373000 & 1.230299000 & 1.263363000 \\
\hline C & 8.393594000 & 0.031593000 & 1.399415000 & $\mathrm{C}$ & 8.387791000 & 0.026561000 & 1.408001000 \\
\hline C & 7.710887000 & -1.175858000 & 1.266138000 & $\mathrm{C}$ & 7.704910000 & -1.180353000 & 1.270584000 \\
\hline C & 5.454536000 & -2.351551000 & 0.824925000 & $\mathrm{C}$ & 5.448521000 & -2.353675000 & 0.820081000 \\
\hline$N$ & 4.164994000 & -2.005211000 & 0.563078000 & $\mathrm{~N}$ & 4.160069000 & -2.006809000 & 0.554273000 \\
\hline C & 3.271118000 & -2.961919000 & 0.375574000 & $\mathrm{C}$ & 3.265108000 & -2.961035000 & 0.359607000 \\
\hline C & 3.574337000 & -4.307492000 & 0.440710000 & $\mathrm{C}$ & 3.567481000 & -4.306703000 & 0.421552000 \\
\hline C & 4.880407000 & -4.705475000 & 0.712179000 & $\mathrm{C}$ & 4.872221000 & -4.706167000 & 0.697706000 \\
\hline C & 5.814093000 & -3.690518000 & 0.902004000 & $\mathrm{C}$ & 5.806424000 & -3.692896000 & 0.895087000 \\
\hline $\mathrm{F}$ & 8.410026000 & 2.329511000 & 1.387277000 & $\mathrm{~F}$ & 8.405545000 & 2.324419000 & 1.398265000 \\
\hline $\mathrm{F}$ & 8.419748000 & -2.266645000 & 1.409150000 & $\mathrm{~F}$ & 8.412255000 & -2.271597000 & 1.412033000 \\
\hline $\mathrm{O}$ & 9.696772000 & 0.035969000 & 1.703056000 & $\mathrm{O}$ & 9.689830000 & 0.029435000 & 1.714338000 \\
\hline C & 10.543287000 & 0.031360000 & 0.596125000 & $\mathrm{C}$ & 10.539326000 & 0.026500000 & 0.609251000 \\
\hline C & -3.579770000 & 4.311756000 & -0.429852000 & $\mathrm{C}$ & -3.565884000 & 4.308519000 & -0.424162000 \\
\hline C & -3.275813000 & 2.966135000 & -0.369333000 & $\mathrm{C}$ & -3.264539000 & 2.962694000 & -0.360847000 \\
\hline C & -4.885957000 & 4.709966000 & -0.700408000 & $\mathrm{C}$ & -4.870044000 & 4.708702000 & -0.702029000 \\
\hline C & -5.818985000 & 3.695163000 & -0.894367000 & $\mathrm{C}$ & -5.804813000 & 3.695963000 & -0.899475000 \\
\hline C & -5.458714000 & 2.356122000 & -0.821896000 & $\mathrm{C}$ & -5.447975000 & 2.356547000 & -0.822995000 \\
\hline$N$ & -4.169108000 & 2.009618000 & -0.560544000 & $\mathrm{~N}$ & -4.160015000 & 2.008987000 & -0.555696000 \\
\hline C & -6.349801000 & 1.217925000 & -1.001980000 & $\mathrm{C}$ & -6.340882000 & 1.219555000 & -1.003958000 \\
\hline C & -5.710018000 & -0.018031000 & -0.872391000 & $\mathrm{C}$ & -5.706015000 & -0.017172000 & -0.870337000 \\
\hline C & -6.342561000 & -1.258424000 & -0.999364000 & $\mathrm{C}$ & -6.338490000 & -1.256484000 & -0.995297000 \\
\hline C & -7.713835000 & 1.180603000 & -1.269883000 & $\mathrm{C}$ & -7.704756000 & 1.184490000 & -1.274898000 \\
\hline C & -8.395568000 & -0.026765000 & -1.408703000 & $\mathrm{C}$ & -8.388251000 & -0.022049000 & -1.412475000 \\
\hline C & -7.707107000 & -1.229746000 & -1.267391000 & $\mathrm{C}$ & -7.702753000 & -1.226163000 & -1.266549000 \\
\hline C & -5.444032000 & -2.389655000 & -0.816099000 & $\mathrm{C}$ & -5.442324000 & -2.388914000 & -0.806117000 \\
\hline$N$ & -4.160570000 & -2.035532000 & -0.553392000 & $\mathrm{~N}$ & -4.159008000 & -2.037222000 & -0.540021000 \\
\hline C & -3.259469000 & -2.988391000 & -0.357562000 & $\mathrm{C}$ & -3.259091000 & -2.989643000 & -0.336745000 \\
\hline
\end{tabular}




\begin{tabular}{|c|c|c|c|c|c|c|c|}
\hline C & -3.557945000 & -4.330853000 & -0.417477000 & $C$ & -3.560007000 & -4.331475000 & -0.392831000 \\
\hline C & -4.864559000 & -4.739418000 & -0.693322000 & $\mathrm{C}$ & -4.866380000 & -4.738513000 & -0.672710000 \\
\hline C & -5.801042000 & -3.734173000 & -0.889795000 & $\mathrm{C}$ & -5.800963000 & -3.732842000 & -0.876810000 \\
\hline $\mathrm{F}$ & -8.423128000 & 2.271457000 & -1.410188000 & $\mathrm{~F}$ & -8.411204000 & 2.276111000 & -1.417670000 \\
\hline $\mathrm{F}$ & -8.410480000 & -2.324735000 & -1.405333000 & $\mathrm{~F}$ & -8.407439000 & -2.319886000 & -1.401825000 \\
\hline $\mathrm{O}$ & -9.698256000 & -0.030734000 & -1.714428000 & $\mathrm{O}$ & -9.689919000 & -0.024151000 & -1.720303000 \\
\hline C & -10.546494000 & -0.031676000 & -0.608807000 & $\mathrm{C}$ & -10.540733000 & -0.021209000 & -0.616222000 \\
\hline $\mathrm{H}$ & 6.187125000 & 0.013894000 & -1.727267000 & $\mathrm{H}$ & 6.183725000 & 0.027258000 & -1.711294000 \\
\hline C & 6.512338000 & -0.042667000 & -4.366275000 & $\mathrm{C}$ & 6.488227000 & -0.040922000 & -4.384674000 \\
\hline $\mathrm{H}$ & 1.865049000 & -0.012509000 & -4.203104000 & $\mathrm{H}$ & 1.856834000 & -0.019509000 & -4.208802000 \\
\hline $\mathrm{H}$ & -1.869525000 & 0.004834000 & 4.200793000 & $\mathrm{H}$ & -1.858980000 & 0.019965000 & 4.208654000 \\
\hline C & -6.492429000 & 0.009709000 & 4.404502000 & $\mathrm{C}$ & -6.481345000 & 0.021146000 & 4.399331000 \\
\hline $\mathrm{H}$ & -6.191176000 & -0.020740000 & 1.722289000 & $\mathrm{H}$ & -6.185819000 & -0.017929000 & 1.709695000 \\
\hline $\mathrm{H}$ & 2.749256000 & 5.057060000 & 0.224923000 & $\mathrm{H}$ & 2.749593000 & 5.055753000 & 0.215016000 \\
\hline $\mathrm{H}$ & 2.233533000 & 2.651174000 & 0.132051000 & $\mathrm{H}$ & 2.233188000 & 2.648823000 & 0.119403000 \\
\hline C & 5.210182000 & 6.222203000 & 0.739562000 & $\mathrm{C}$ & 5.207902000 & 6.219132000 & 0.743790000 \\
\hline $\mathrm{H}$ & 6.845119000 & 3.983247000 & 1.085728000 & $\mathrm{H}$ & 6.840947000 & 3.980360000 & 1.097030000 \\
\hline $\mathrm{H}$ & 2.244550000 & -2.627961000 & 0.160884000 & $\mathrm{H}$ & 2.240040000 & -2.624336000 & 0.142463000 \\
\hline $\mathrm{H}$ & 2.768307000 & -5.030514000 & 0.273938000 & $\mathrm{H}$ & 2.761951000 & -5.028778000 & 0.248860000 \\
\hline C & 5.306779000 & -6.162143000 & 0.799736000 & $\mathrm{C}$ & 5.296822000 & -6.163366000 & 0.782300000 \\
\hline $\mathrm{H}$ & 6.861286000 & -3.932674000 & 1.117063000 & $\mathrm{H}$ & 6.852349000 & -3.936866000 & 1.114050000 \\
\hline $\mathrm{H}$ & 10.394088000 & 0.926669000 & -0.040371000 & $\mathrm{H}$ & 10.392449000 & 0.923414000 & -0.025385000 \\
\hline $\mathrm{H}$ & 10.399891000 & -0.873214000 & -0.028517000 & $\mathrm{H}$ & 10.396496000 & -0.876571000 & -0.017551000 \\
\hline $\mathrm{H}$ & 11.578173000 & 0.037061000 & 0.973100000 & $\mathrm{H}$ & 11.573073000 & 0.030386000 & 0.989143000 \\
\hline $\mathrm{H}$ & -2.774208000 & 5.034613000 & -0.260163000 & $\mathrm{H}$ & -2.760016000 & 5.030150000 & -0.251209000 \\
\hline $\mathrm{H}$ & -2.249143000 & 2.631993000 & -0.155436000 & $\mathrm{H}$ & -2.239936000 & 2.625418000 & -0.142417000 \\
\hline C & -5.313175000 & 6.166704000 & -0.782445000 & C & -5.293400000 & 6.166145000 & -0.788620000 \\
\hline $\mathrm{H}$ & -6.866219000 & 3.937515000 & -1.109028000 & $\mathrm{H}$ & -6.850323000 & 3.940520000 & -1.119763000 \\
\hline $\mathrm{H}$ & -2.235774000 & -2.647015000 & -0.141755000 & $\mathrm{H}$ & -2.236075000 & -2.647759000 & -0.118912000 \\
\hline $\mathrm{H}$ & -2.749614000 & -5.052926000 & -0.245137000 & $\mathrm{H}$ & -2.753836000 & -5.054408000 & -0.214575000 \\
\hline C & -5.208747000 & -6.217736000 & -0.768795000 & C & -5.212598000 & -6.216382000 & -0.744266000 \\
\hline $\mathrm{H}$ & -6.844875000 & -3.978583000 & -1.108176000 & $\mathrm{H}$ & -6.844193000 & -3.976732000 & -1.098475000 \\
\hline $\mathrm{H}$ & -10.404120000 & 0.869804000 & 0.020518000 & $\mathrm{H}$ & -10.397698000 & 0.881215000 & 0.011456000 \\
\hline $\mathrm{H}$ & -10.398185000 & -0.930082000 & 0.023516000 & $\mathrm{H}$ & -10.395550000 & -0.918780000 & 0.017871000 \\
\hline $\mathrm{H}$ & -11.580796000 & -0.035647000 & -0.987403000 & $\mathrm{H}$ & -11.574023000 & -0.023754000 & -0.997359000 \\
\hline C & 4.138060000 & -7.103009000 & 0.581384000 & C & 4.128271000 & -7.102464000 & 0.555634000 \\
\hline C & 6.360085000 & -6.428585000 & -0.268136000 & C & 6.354538000 & -6.426678000 & -0.282049000 \\
\hline C & 5.896395000 & -6.422083000 & 2.180077000 & $\mathrm{C}$ & 5.880183000 & -6.428013000 & 2.164430000 \\
\hline C & 4.376131000 & 6.866689000 & 1.839561000 & C & 4.368142000 & 6.862497000 & 1.840151000 \\
\hline C & 4.878682000 & 6.865725000 & -0.600920000 & $\mathrm{C}$ & 4.883050000 & 6.863332000 & -0.598023000 \\
\hline C & 6.678555000 & 6.447369000 & 1.041833000 & $\mathrm{C}$ & 6.674755000 & 6.443802000 & 1.053627000 \\
\hline C & -6.366232000 & 6.428466000 & 0.286839000 & $\mathrm{C}$ & -6.352726000 & 6.431304000 & 0.273661000 \\
\hline C & -4.144907000 & 7.107399000 & -0.560928000 & C & -4.124515000 & 7.104561000 & -0.560851000 \\
\hline C & -5.903464000 & 6.431600000 & -2.161549000 & C & -5.874177000 & 6.429864000 & -2.172022000 \\
\hline C & -6.676363000 & -6.442765000 & -1.074833000 & C & -6.679462000 & -6.440236000 & -1.054630000 \\
\hline C & -4.372092000 & -6.857089000 & -1.869814000 & $\mathrm{C}$ & -4.372787000 & -6.860316000 & -1.840256000 \\
\hline C & -4.879331000 & -6.866521000 & 0.569662000 & $\mathrm{C}$ & -4.888611000 & -6.860625000 & 0.597739000 \\
\hline $\mathrm{H}$ & 3.682049000 & -6.983772000 & -0.418850000 & $\mathrm{H}$ & 3.676913000 & -6.979639000 & -0.446255000 \\
\hline $\mathrm{H}$ & 3.342704000 & -6.968866000 & 1.337818000 & $\mathrm{H}$ & 3.329736000 & -6.970312000 & 1.309045000 \\
\hline $\mathrm{H}$ & 4.481215000 & -8.150446000 & 0.655998000 & $\mathrm{H}$ & 4.470401000 & -8.150333000 & 0.628297000 \\
\hline $\mathrm{H}$ & 7.262529000 & -5.804503000 & -0.136783000 & $\mathrm{H}$ & 7.257001000 & -5.803964000 & -0.144686000 \\
\hline $\mathrm{H}$ & 5.966695000 & -6.236788000 & -1.283675000 & $\mathrm{H}$ & 5.965639000 & -6.231146000 & -1.298577000 \\
\hline $\mathrm{H}$ & 6.685878000 & -7.484774000 & -0.230288000 & $\mathrm{H}$ & 6.679080000 & -7.483278000 & -0.246277000 \\
\hline $\mathrm{H}$ & 5.159056000 & -6.226783000 & 2.980680000 & $\mathrm{H}$ & 5.139463000 & -6.234998000 & 2.962430000 \\
\hline $\mathrm{H}$ & 6.784854000 & -5.798101000 & 2.385735000 & $\mathrm{H}$ & 6.768259000 & -5.805526000 & 2.376072000 \\
\hline $\mathrm{H}$ & 6.213098000 & -7.478013000 & 2.268188000 & $\mathrm{H}$ & 6.195652000 & -7.484467000 & 2.250143000 \\
\hline $\mathrm{H}$ & 4.586404000 & 6.414167000 & 2.826376000 & $\mathrm{H}$ & 4.573339000 & 6.409138000 & 2.827628000 \\
\hline $\mathrm{H}$ & 3.290375000 & 6.775414000 & 1.655243000 & $\mathrm{H}$ & 3.283345000 & 6.771706000 & 1.650166000 \\
\hline $\mathrm{H}$ & 4.604827000 & 7.946311000 & 1.911499000 & $\mathrm{H}$ & 4.596807000 & 7.941951000 & 1.914061000 \\
\hline $\mathrm{H}$ & 3.807701000 & 6.778640000 & -0.858940000 & $\mathrm{H}$ & 3.813326000 & 6.777000000 & -0.861353000 \\
\hline $\mathrm{H}$ & 5.458850000 & 6.410378000 & -1.424866000 & $\mathrm{H}$ & 5.467134000 & 6.408375000 & -1.419381000 \\
\hline $\mathrm{H}$ & 5.121297000 & 7.944465000 & -0.578273000 & $\mathrm{H}$ & 5.126020000 & 7.941903000 & -0.573280000 \\
\hline $\mathrm{H}$ & 7.339222000 & 6.020110000 & 0.264957000 & $\mathrm{H}$ & 7.339267000 & 6.017181000 & 0.279700000 \\
\hline $\mathrm{H}$ & 6.978211000 & 6.021403000 & 2.017155000 & $\mathrm{H}$ & 6.969442000 & 6.017236000 & 2.030186000 \\
\hline $\mathrm{H}$ & 6.890362000 & 7.530734000 & 1.084844000 & $\mathrm{H}$ & 6.886338000 & 7.527099000 & 1.098473000 \\
\hline
\end{tabular}




\begin{tabular}{|c|c|c|c|c|c|c|c|}
\hline $\mathrm{H}$ & -5.972276000 & 6.233176000 & 1.301494000 & $\mathrm{H}$ & -5.965708000 & 6.236492000 & 1.291044000 \\
\hline $\mathrm{H}$ & -7.268291000 & 5.804241000 & 0.153518000 & $\mathrm{H}$ & -7.255458000 & 5.809187000 & 0.135387000 \\
\hline $\mathrm{H}$ & -6.692763000 & 7.484562000 & 0.253044000 & $\mathrm{H}$ & -6.676368000 & 7.488123000 & 0.236292000 \\
\hline $\mathrm{H}$ & -3.349669000 & 6.976435000 & -1.318044000 & $\mathrm{H}$ & -3.324878000 & 6.971226000 & -1.312880000 \\
\hline $\mathrm{H}$ & -3.688599000 & 6.984749000 & 0.438757000 & $\mathrm{H}$ & -3.674854000 & 6.982207000 & 0.441858000 \\
\hline $\mathrm{H}$ & -4.488634000 & 8.154919000 & -0.631593000 & $\mathrm{H}$ & -4.465763000 & 8.152618000 & -0.634926000 \\
\hline $\mathrm{H}$ & -6.791565000 & 5.807827000 & -2.369348000 & $\mathrm{H}$ & -6.762338000 & 5.807806000 & -2.384574000 \\
\hline $\mathrm{H}$ & -5.166270000 & 6.240003000 & -2.963177000 & $\mathrm{H}$ & -5.132218000 & 6.235500000 & -2.968542000 \\
\hline $\mathrm{H}$ & -6.220941000 & 7.487645000 & -2.245363000 & $\mathrm{H}$ & -6.188709000 & 7.486466000 & -2.259333000 \\
\hline $\mathrm{H}$ & -6.974484000 & -6.012847000 & -2.048891000 & $\mathrm{H}$ & -6.973539000 & -6.013610000 & -2.031346000 \\
\hline $\mathrm{H}$ & -7.338852000 & -6.019359000 & -0.297397000 & $\mathrm{H}$ & -7.344019000 & -6.013162000 & -0.280991000 \\
\hline $\mathrm{H}$ & -6.887232000 & -7.526102000 & -1.122875000 & $\mathrm{H}$ & -6.891642000 & -7.523416000 & -1.099442000 \\
\hline $\mathrm{H}$ & -3.286761000 & -6.765705000 & -1.683073000 & $\mathrm{H}$ & -3.288011000 & -6.770093000 & -1.649894000 \\
\hline $\mathrm{H}$ & -4.580876000 & -6.400772000 & -2.855196000 & $\mathrm{H}$ & -4.577389000 & -6.406962000 & -2.827858000 \\
\hline $\mathrm{H}$ & -4.599784000 & -7.936593000 & -1.946525000 & $\mathrm{H}$ & -4.602014000 & -7.939651000 & -1.914118000 \\
\hline $\mathrm{H}$ & -5.461600000 & -6.415193000 & 1.394338000 & $\mathrm{H}$ & -5.472707000 & -6.405225000 & 1.418841000 \\
\hline $\mathrm{H}$ & -3.808957000 & -6.779503000 & 0.830207000 & $\mathrm{H}$ & -3.818928000 & -6.774928000 & 0.861443000 \\
\hline $\mathrm{H}$ & -5.120860000 & -7.945387000 & 0.542009000 & $\mathrm{H}$ & -5.132233000 & -7.939047000 & 0.573020000 \\
\hline $\mathrm{F}$ & 6.377513000 & 0.205506000 & -5.645894000 & $\mathrm{~F}$ & 6.418826000 & 0.635405000 & -5.509386000 \\
\hline $\mathrm{F}$ & 7.363264000 & 0.834932000 & -3.884729000 & $\mathrm{~F}$ & 7.491439000 & 0.448133000 & -3.694907000 \\
\hline $\mathrm{F}$ & 7.091753000 & -1.219949000 & -4.261818000 & $\mathrm{~F}$ & 6.823455000 & -1.273814000 & -4.713502000 \\
\hline $\mathrm{F}$ & -6.501796000 & -0.899940000 & 5.353389000 & $\mathrm{~F}$ & -6.499933000 & -0.903985000 & 5.335767000 \\
\hline $\mathrm{F}$ & -6.681665000 & 1.169079000 & 4.998403000 & $\mathrm{~F}$ & -6.659970000 & 1.171487000 & 5.018239000 \\
\hline $\mathrm{H}$ & 4.009494000 & -0.016801000 & -5.444670000 & $\mathrm{H}$ & 3.999812000 & -0.010485000 & -5.431419000 \\
\hline $\mathrm{H}$ & -4.011651000 & -0.007123000 & 5.439581000 & $\mathrm{H}$ & -4.000785000 & 0.013217000 & 5.429939000 \\
\hline $\mathrm{F}$ & -7.537534000 & -0.207603000 & 3.645712000 & $\mathrm{~F}$ & -7.533041000 & -0.171727000 & 3.641803000 \\
\hline
\end{tabular}


Table S8. DFT optimized ground state $\left(\mathrm{S}_{0}\right)$ and $\mathrm{T}_{1}$ state geometry of Ir-2' in cartesian (XYZ) coordinates

\begin{tabular}{|c|c|c|c|c|c|c|c|}
\hline \multicolumn{4}{|c|}{ State $S_{0}$} & \multicolumn{4}{|c|}{ State $T_{1}$} \\
\hline $\mathrm{N}$ & 1.744239000 & 0.004807000 & -0.169899000 & $\mathrm{~N}$ & 1.734640000 & 0.005219000 & -0.142441000 \\
\hline C & 0.546378000 & 0.007263000 & 0.421900000 & C & 0.564333000 & 0.009219000 & 0.433445000 \\
\hline C & -0.546379000 & -0.007264000 & -0.421898000 & $\mathrm{C}$ & -0.564334000 & -0.009217000 & -0.433442000 \\
\hline$S$ & -0.027577000 & -0.025969000 & -2.056894000 & $S$ & -0.052501000 & -0.035116000 & -2.076200000 \\
\hline C & 1.608562000 & -0.011941000 & -1.482718000 & $\mathrm{C}$ & 1.609954000 & -0.017045000 & -1.493316000 \\
\hline$S$ & 0.027577000 & 0.025969000 & 2.056896000 & $S$ & 0.052500000 & 0.035119000 & 2.076204000 \\
\hline C & -1.608562000 & 0.011942000 & 1.482720000 & $\mathrm{C}$ & -1.609955000 & 0.017049000 & 1.493320000 \\
\hline $\mathrm{N}$ & -1.744240000 & -0.004806000 & 0.169901000 & $\mathrm{~N}$ & -1.734641000 & -0.005215000 & 0.142445000 \\
\hline C & -2.791318000 & 0.016562000 & 2.299866000 & $\mathrm{C}$ & -2.763390000 & 0.022659000 & 2.288484000 \\
\hline C & 2.791318000 & -0.016562000 & -2.299863000 & $\mathrm{C}$ & 2.763389000 & -0.022658000 & -2.288480000 \\
\hline C & 4.009670000 & -0.002267000 & -1.580545000 & $\mathrm{C}$ & 4.008269000 & -0.004281000 & -1.571731000 \\
\hline C & 5.170644000 & -0.005076000 & -2.354054000 & $\mathrm{C}$ & 5.163169000 & -0.006900000 & -2.344423000 \\
\hline C & 5.124038000 & -0.021816000 & -3.739305000 & $\mathrm{C}$ & 5.116609000 & -0.027543000 & -3.732542000 \\
\hline C & 3.916320000 & -0.036809000 & -4.449182000 & $\mathrm{C}$ & 3.897566000 & -0.047110000 & -4.440789000 \\
\hline C & 2.751623000 & -0.033566000 & -3.698408000 & $\mathrm{C}$ & 2.733607000 & -0.044137000 & -3.701828000 \\
\hline C & -2.751624000 & 0.033564000 & 3.698410000 & $\mathrm{C}$ & -2.733607000 & 0.044136000 & 3.701831000 \\
\hline C & -3.916321000 & 0.036805000 & 4.449184000 & $\mathrm{C}$ & -3.897567000 & 0.047106000 & 4.440793000 \\
\hline C & -5.124039000 & 0.021811000 & 3.739307000 & $\mathrm{C}$ & -5.116609000 & 0.027539000 & 3.732545000 \\
\hline C & -5.170645000 & 0.005073000 & 2.354056000 & $\mathrm{C}$ & -5.163169000 & 0.006897000 & 2.344426000 \\
\hline C & -4.009671000 & 0.002266000 & 1.580547000 & $\mathrm{C}$ & -4.008269000 & 0.004281000 & 1.571734000 \\
\hline Ir & -3.842532000 & -0.015182000 & -0.423159000 & Ir & -3.835905000 & -0.016574000 & -0.420738000 \\
\hline $\mathrm{Cl}$ & -3.255832000 & -0.031943000 & -2.848315000 & $\mathrm{Cl}$ & -3.281688000 & -0.036949000 & -2.848554000 \\
\hline Ir & 3.842532000 & 0.015184000 & 0.423161000 & Ir & 3.835905000 & 0.016577000 & 0.420742000 \\
\hline C & 3.925016000 & -0.055523000 & -5.973217000 & $\mathrm{C}$ & 3.912828000 & -0.070180000 & -5.963646000 \\
\hline C & 4.657312000 & -1.301010000 & -6.455892000 & $\mathrm{C}$ & 4.651293000 & -1.315656000 & -6.437790000 \\
\hline C & 4.642703000 & 1.186242000 & -6.486374000 & $\mathrm{C}$ & 4.630956000 & 1.172233000 & -6.475452000 \\
\hline C & 2.521147000 & -0.070889000 & -6.546702000 & $\mathrm{C}$ & 2.512829000 & -0.090553000 & -6.545865000 \\
\hline C & -3.925017000 & 0.055519000 & 5.973219000 & $\mathrm{C}$ & -3.912828000 & 0.070172000 & 5.963650000 \\
\hline C & -2.521148000 & 0.070882000 & 6.546704000 & $\mathrm{C}$ & -2.512830000 & 0.090545000 & 6.545869000 \\
\hline C & -4.642707000 & -1.186244000 & 6.486377000 & $\mathrm{C}$ & -4.630955000 & -1.172243000 & 6.475453000 \\
\hline C & -4.657310000 & 1.301008000 & 6.455893000 & $\mathrm{C}$ & -4.651295000 & 1.315646000 & 6.437797000 \\
\hline $\mathrm{Cl}$ & 3.255833000 & 0.031947000 & 2.848317000 & $\mathrm{Cl}$ & 3.281687000 & 0.036952000 & 2.848557000 \\
\hline C & 3.569315000 & 4.331675000 & 0.314462000 & $\mathrm{C}$ & 3.562434000 & 4.332260000 & 0.305578000 \\
\hline C & 3.268876000 & 2.988761000 & 0.274473000 & $\mathrm{C}$ & 3.262003000 & 2.989455000 & 0.266293000 \\
\hline C & 4.881039000 & 4.742109000 & 0.562216000 & $\mathrm{C}$ & 4.874237000 & 4.742717000 & 0.553381000 \\
\hline C & 5.820276000 & 3.738221000 & 0.752030000 & $\mathrm{C}$ & 5.814176000 & 3.739432000 & 0.743572000 \\
\hline C & 5.461175000 & 2.393080000 & 0.698789000 & $\mathrm{C}$ & 5.455610000 & 2.394340000 & 0.690364000 \\
\hline$N$ & 4.172601000 & 2.037137000 & 0.464108000 & $\mathrm{~N}$ & 4.166915000 & 2.039172000 & 0.456114000 \\
\hline C & 6.362361000 & 1.262949000 & 0.874329000 & $\mathrm{C}$ & 6.357219000 & 1.264191000 & 0.866434000 \\
\hline C & 5.725772000 & 0.021995000 & 0.766650000 & $\mathrm{C}$ & 5.722127000 & 0.023313000 & 0.761061000 \\
\hline C & 6.368579000 & -1.213888000 & 0.889314000 & $\mathrm{C}$ & 6.362811000 & -1.212502000 & 0.884783000 \\
\hline C & 7.731457000 & 1.234643000 & 1.116162000 & $\mathrm{C}$ & 7.726774000 & 1.236122000 & 1.107275000 \\
\hline C & 8.421992000 & 0.031977000 & 1.250698000 & $\mathrm{C}$ & 8.416843000 & 0.033286000 & 1.242880000 \\
\hline C & 7.737163000 & -1.175764000 & 1.130653000 & $\mathrm{C}$ & 7.731887000 & -1.174613000 & 1.125019000 \\
\hline C & 5.474083000 & -2.351982000 & 0.727795000 & $\mathrm{C}$ & 5.467467000 & -2.350714000 & 0.725593000 \\
\hline$N$ & 4.179529000 & -2.005127000 & 0.491782000 & $\mathrm{~N}$ & 4.172957000 & -2.004763000 & 0.489122000 \\
\hline C & 3.283159000 & -2.961650000 & 0.315531000 & $\mathrm{C}$ & 3.275082000 & -2.960044000 & 0.314934000 \\
\hline C & 3.588401000 & -4.307485000 & 0.368140000 & $\mathrm{C}$ & 3.579848000 & -4.305764000 & 0.370034000 \\
\hline C & 4.899506000 & -4.705927000 & 0.613693000 & $\mathrm{C}$ & 4.890862000 & -4.704078000 & 0.616668000 \\
\hline C & 5.835757000 & -3.691269000 & 0.791757000 & $\mathrm{C}$ & 5.828158000 & -3.689914000 & 0.792835000 \\
\hline $\mathrm{F}$ & 8.438729000 & 2.330192000 & 1.235050000 & $\mathrm{~F}$ & 8.434183000 & 2.331464000 & 1.222930000 \\
\hline $\mathrm{F}$ & 8.449438000 & -2.266549000 & 1.262587000 & $\mathrm{~F}$ & 8.443778000 & -2.265225000 & 1.256584000 \\
\hline $\mathrm{O}$ & 9.730666000 & 0.036747000 & 1.532887000 & $\mathrm{O}$ & 9.725558000 & 0.038087000 & 1.523332000 \\
\hline C & 10.558374000 & 0.032092000 & 0.412483000 & $\mathrm{C}$ & 10.552285000 & 0.031851000 & 0.401944000 \\
\hline C & -3.588411000 & 4.307487000 & -0.368108000 & $\mathrm{C}$ & -3.579850000 & 4.305767000 & -0.370022000 \\
\hline C & -3.283165000 & 2.961653000 & -0.315513000 & $\mathrm{C}$ & -3.275083000 & 2.960047000 & -0.314924000 \\
\hline C & -4.899517000 & 4.705928000 & -0.613654000 & $\mathrm{C}$ & -4.890864000 & 4.704080000 & -0.616658000 \\
\hline C & -5.835766000 & 3.691270000 & -0.791726000 & $\mathrm{C}$ & -5.828159000 & 3.689917000 & -0.792826000 \\
\hline C & -5.474089000 & 2.351984000 & -0.727778000 & $\mathrm{C}$ & -5.467467000 & 2.350717000 & -0.725586000 \\
\hline$N$ & -4.179534000 & 2.005130000 & -0.491771000 & $\mathrm{~N}$ & -4.172958000 & 2.004766000 & -0.489115000 \\
\hline C & -6.368582000 & 1.213888000 & -0.889305000 & $\mathrm{C}$ & -6.362812000 & 1.212505000 & -0.884777000 \\
\hline
\end{tabular}




\begin{tabular}{|c|c|c|c|c|c|c|c|}
\hline C & -5.725772000 & -0.021993000 & -0.766649000 & C & -5.722127000 & -0.023310000 & -0.761058000 \\
\hline C & -6.362358000 & -1.262948000 & -0.874336000 & $\mathrm{C}$ & -6.357219000 & -1.264188000 & -0.866435000 \\
\hline C & -7.737167000 & 1.175763000 & -1.130643000 & $\mathrm{C}$ & -7.731887000 & 1.174616000 & -1.125013000 \\
\hline C & -8.421993000 & -0.031979000 & -1.250695000 & $\mathrm{C}$ & -8.416843000 & -0.033283000 & -1.242879000 \\
\hline C & -7.731454000 & -1.234644000 & -1.116167000 & $\mathrm{C}$ & -7.726773000 & -1.236120000 & -1.107279000 \\
\hline C & -5.461169000 & -2.393079000 & -0.698806000 & $\mathrm{C}$ & -5.455609000 & -2.394337000 & -0.690369000 \\
\hline$N$ & -4.172596000 & -2.037135000 & -0.464117000 & $\mathrm{~N}$ & -4.166914000 & -2.039170000 & -0.456116000 \\
\hline C & -3.268870000 & -2.988759000 & -0.274491000 & $\mathrm{C}$ & -3.262001000 & -2.989452000 & -0.266300000 \\
\hline C & -3.569304000 & -4.331673000 & -0.314499000 & $\mathrm{C}$ & -3.562431000 & -4.332258000 & -0.305595000 \\
\hline C & -4.881026000 & -4.742108000 & -0.562263000 & $\mathrm{C}$ & -4.874232000 & -4.742715000 & -0.553402000 \\
\hline C & -5.820266000 & -3.738221000 & -0.752065000 & $\mathrm{C}$ & -5.814173000 & -3.739429000 & -0.743587000 \\
\hline $\mathrm{F}$ & -8.449444000 & 2.266547000 & -1.262568000 & $\mathrm{~F}$ & -8.443780000 & 2.265228000 & -1.256573000 \\
\hline $\mathrm{F}$ & -8.438724000 & -2.330194000 & -1.235062000 & $\mathrm{~F}$ & -8.434181000 & -2.331462000 & -1.222941000 \\
\hline $\mathrm{O}$ & -9.730667000 & -0.036749000 & -1.532883000 & $\mathrm{O}$ & -9.725558000 & -0.038079000 & -1.523331000 \\
\hline C & -10.558373000 & -0.032111000 & -0.412477000 & $\mathrm{C}$ & -10.552285000 & -0.031912000 & -0.401942000 \\
\hline $\mathrm{H}$ & 6.156606000 & 0.005838000 & -1.861932000 & $\mathrm{H}$ & 6.149204000 & 0.007082000 & -1.852739000 \\
\hline $\mathrm{H}$ & 6.076941000 & -0.023488000 & -4.291864000 & $\mathrm{H}$ & 6.066404000 & -0.029015000 & -4.288175000 \\
\hline $\mathrm{H}$ & 1.771769000 & -0.044695000 & -4.200143000 & $\mathrm{H}$ & 1.756041000 & -0.058703000 & -4.206329000 \\
\hline $\mathrm{H}$ & -1.771770000 & 0.044694000 & 4.200145000 & $\mathrm{H}$ & -1.756042000 & 0.058701000 & 4.206333000 \\
\hline $\mathrm{H}$ & -6.076942000 & 0.023481000 & 4.291865000 & $\mathrm{H}$ & -6.066405000 & 0.029009000 & 4.288178000 \\
\hline $\mathrm{H}$ & -6.156607000 & -0.005842000 & 1.861933000 & $\mathrm{H}$ & -6.149205000 & -0.007084000 & 1.852743000 \\
\hline $\mathrm{H}$ & 4.160059000 & -2.224228000 & -6.103520000 & $\mathrm{H}$ & 4.154215000 & -2.238743000 & -6.084878000 \\
\hline $\mathrm{H}$ & 5.705444000 & -1.335773000 & -6.106476000 & $\mathrm{H}$ & 5.698031000 & -1.347238000 & -6.084288000 \\
\hline $\mathrm{H}$ & 4.682056000 & -1.336688000 & -7.561795000 & $\mathrm{H}$ & 4.680322000 & -1.354255000 & -7.543482000 \\
\hline $\mathrm{H}$ & 5.689793000 & 1.242590000 & -6.136595000 & $\mathrm{H}$ & 5.676296000 & 1.232498000 & -6.121481000 \\
\hline $\mathrm{H}$ & 4.133689000 & 2.111870000 & -6.157903000 & $\mathrm{H}$ & 4.117662000 & 2.097262000 & -6.152045000 \\
\hline $\mathrm{H}$ & 4.668680000 & 1.194363000 & -7.592800000 & $\mathrm{H}$ & 4.661495000 & 1.176929000 & -7.581773000 \\
\hline $\mathrm{H}$ & 1.938182000 & 0.823480000 & -6.257461000 & $\mathrm{H}$ & 1.926378000 & 0.803289000 & -6.262506000 \\
\hline $\mathrm{H}$ & 1.948384000 & -0.964195000 & -6.234689000 & $\mathrm{H}$ & 1.940520000 & -0.984111000 & -6.234112000 \\
\hline $\mathrm{H}$ & 2.563862000 & -0.084709000 & -7.651154000 & $\mathrm{H}$ & 2.562754000 & -0.107681000 & -7.649962000 \\
\hline $\mathrm{H}$ & -1.948383000 & 0.964187000 & 6.234689000 & $\mathrm{H}$ & -1.940522000 & 0.984106000 & 6.234118000 \\
\hline $\mathrm{H}$ & -1.938186000 & -0.823489000 & 6.257464000 & $\mathrm{H}$ & -1.926378000 & -0.803294000 & 6.262507000 \\
\hline $\mathrm{H}$ & -2.563863000 & 0.084704000 & 7.651156000 & $\mathrm{H}$ & -2.562755000 & 0.107671000 & 7.649966000 \\
\hline $\mathrm{H}$ & -4.133694000 & -2.111873000 & 6.157907000 & $\mathrm{H}$ & -4.117659000 & -2.097270000 & 6.152044000 \\
\hline $\mathrm{H}$ & -5.689796000 & -1.242590000 & 6.136600000 & $\mathrm{H}$ & -5.676294000 & -1.232509000 & 6.121482000 \\
\hline $\mathrm{H}$ & -4.668682000 & -1.194364000 & 7.592804000 & $\mathrm{H}$ & -4.661494000 & -1.176941000 & 7.581774000 \\
\hline $\mathrm{H}$ & -5.705443000 & 1.335772000 & 6.106478000 & $\mathrm{H}$ & -5.698033000 & 1.347228000 & 6.084294000 \\
\hline $\mathrm{H}$ & -4.160057000 & 2.224225000 & 6.103519000 & $\mathrm{H}$ & -4.154218000 & 2.238735000 & 6.084888000 \\
\hline $\mathrm{H}$ & -4.682054000 & 1.336688000 & 7.561796000 & $\mathrm{H}$ & -4.680325000 & 1.354242000 & 7.543489000 \\
\hline $\mathrm{H}$ & 2.758518000 & 5.052716000 & 0.149092000 & $\mathrm{H}$ & 2.751719000 & 5.053235000 & 0.139877000 \\
\hline $\mathrm{H}$ & 2.241425000 & 2.644924000 & 0.081195000 & $\mathrm{H}$ & 2.235001000 & 2.643989000 & 0.073668000 \\
\hline C & 5.227524000 & 6.221004000 & 0.615516000 & $\mathrm{C}$ & 5.220267000 & 6.221630000 & 0.606496000 \\
\hline $\mathrm{H}$ & 6.868216000 & 3.983925000 & 0.948327000 & $\mathrm{H}$ & 6.861920000 & 3.985762000 & 0.940006000 \\
\hline $\mathrm{H}$ & 2.252883000 & -2.626365000 & 0.121305000 & $\mathrm{H}$ & 2.245354000 & -2.623305000 & 0.120499000 \\
\hline $\mathrm{H}$ & 2.779963000 & -5.030357000 & 0.212370000 & $\mathrm{H}$ & 2.771286000 & -5.028713000 & 0.215609000 \\
\hline C & 5.328756000 & -6.162790000 & 0.684655000 & $\mathrm{C}$ & 5.319088000 & -6.160988000 & 0.690923000 \\
\hline $\mathrm{H}$ & 6.886986000 & -3.933773000 & 0.985858000 & $\mathrm{H}$ & 6.879081000 & -3.932904000 & 0.987804000 \\
\hline $\mathrm{H}$ & 10.399504000 & 0.927760000 & -0.221354000 & $\mathrm{H}$ & 10.392972000 & 0.926834000 & -0.232659000 \\
\hline $\mathrm{H}$ & 10.404279000 & -0.872066000 & -0.210402000 & $\mathrm{H}$ & 10.397266000 & -0.873049000 & -0.219545000 \\
\hline $\mathrm{H}$ & 11.599596000 & 0.036974000 & 0.771883000 & $\mathrm{H}$ & 11.593733000 & 0.036880000 & 0.760530000 \\
\hline $\mathrm{H}$ & -2.779975000 & 5.030360000 & -0.212332000 & $\mathrm{H}$ & -2.771289000 & 5.028716000 & -0.215595000 \\
\hline $\mathrm{H}$ & -2.252889000 & 2.626368000 & -0.121294000 & $\mathrm{H}$ & -2.245356000 & 2.623309000 & -0.120488000 \\
\hline C & -5.328771000 & 6.162791000 & -0.684599000 & $\mathrm{C}$ & -5.319090000 & 6.160991000 & -0.690911000 \\
\hline $\mathrm{H}$ & -6.886997000 & 3.933773000 & -0.985823000 & $\mathrm{H}$ & -6.879082000 & 3.932906000 & -0.987796000 \\
\hline $\mathrm{H}$ & -2.241421000 & -2.644922000 & -0.081203000 & $\mathrm{H}$ & -2.235000000 & -2.643986000 & -0.073671000 \\
\hline $\mathrm{H}$ & -2.758505000 & -5.052715000 & -0.149137000 & $\mathrm{H}$ & -2.751715000 & -5.053233000 & -0.139899000 \\
\hline C & -5.227506000 & -6.221003000 & -0.615587000 & $\mathrm{C}$ & -5.220261000 & -6.221628000 & -0.606528000 \\
\hline $\mathrm{H}$ & -6.868205000 & -3.983925000 & -0.948368000 & $\mathrm{H}$ & -6.861917000 & -3.985760000 & -0.940023000 \\
\hline $\mathrm{H}$ & -10.404270000 & 0.872035000 & 0.210426000 & $\mathrm{H}$ & -10.397271000 & 0.872955000 & 0.219596000 \\
\hline $\mathrm{H}$ & -10.399508000 & -0.927791000 & 0.221342000 & $\mathrm{H}$ & -10.392967000 & -0.926929000 & 0.232611000 \\
\hline $\mathrm{H}$ & -11.599596000 & -0.036977000 & -0.771876000 & $\mathrm{H}$ & -11.593733000 & -0.036929000 & -0.760529000 \\
\hline C & 4.156911000 & -7.103618000 & 0.483574000 & $\mathrm{C}$ & 4.146821000 & -7.101536000 & 0.491108000 \\
\hline C & 6.361297000 & -6.422322000 & -0.404984000 & $\mathrm{C}$ & 6.352131000 & -6.423101000 & -0.397655000 \\
\hline C & 5.945453000 & -6.430729000 & 2.051522000 & $\mathrm{C}$ & 5.934805000 & -6.426066000 & 2.058808000 \\
\hline C & 4.411739000 & 6.873266000 & 1.724569000 & $\mathrm{C}$ & 4.404133000 & 6.873438000 & 1.715593000 \\
\hline
\end{tabular}




\begin{tabular}{|c|c|c|c|c|c|c|c|}
\hline C & 4.874433000 & 6.855870000 & -0.723547000 & $\mathrm{C}$ & 4.866736000 & 6.855942000 & -0.732733000 \\
\hline C & 6.700599000 & 6.448261000 & 0.892372000 & $\mathrm{C}$ & 6.693265000 & 6.449435000 & 0.883253000 \\
\hline C & -6.361308000 & 6.422308000 & 0.405047000 & C & -6.352136000 & 6.423102000 & 0.397665000 \\
\hline C & -4.156928000 & 7.103619000 & -0.483513000 & C & -4.146825000 & 7.101540000 & -0.491094000 \\
\hline C & -5.945475000 & 6.430744000 & -2.051461000 & $\mathrm{C}$ & -5.934805000 & 6.426070000 & -2.058797000 \\
\hline C & -6.700580000 & -6.448261000 & -0.892450000 & $\mathrm{C}$ & -6.693258000 & -6.449432000 & -0.883288000 \\
\hline C & -4.411716000 & -6.873246000 & -1.724646000 & C & -4.404124000 & -6.873428000 & -1.715626000 \\
\hline C & -4.874417000 & -6.855888000 & 0.723469000 & C & -4.866732000 & -6.855947000 & 0.732700000 \\
\hline $\mathrm{H}$ & 3.681177000 & -6.978804000 & -0.506766000 & $\mathrm{H}$ & 3.671742000 & -6.978576000 & -0.499764000 \\
\hline $\mathrm{H}$ & 3.376316000 & -6.974662000 & 1.256107000 & $\mathrm{H}$ & 3.365913000 & -6.970723000 & 1.262994000 \\
\hline $\mathrm{H}$ & 4.502295000 & -8.151211000 & 0.545067000 & $\mathrm{H}$ & 4.491740000 & -8.149118000 & 0.554942000 \\
\hline $\mathrm{H}$ & 7.265482000 & -5.797912000 & -0.287534000 & $\mathrm{H}$ & 7.256789000 & -5.799294000 & -0.280787000 \\
\hline $\mathrm{H}$ & 5.947942000 & -6.224946000 & -1.411517000 & $\mathrm{H}$ & 5.939556000 & -6.227335000 & -1.404804000 \\
\hline $\mathrm{H}$ & 6.688901000 & -7.478375000 & -0.379726000 & $\mathrm{H}$ & 6.678766000 & -7.479368000 & -0.370127000 \\
\hline $\mathrm{H}$ & 5.223776000 & -6.240526000 & 2.867498000 & $\mathrm{H}$ & 5.212823000 & -6.233471000 & 2.873932000 \\
\hline $\mathrm{H}$ & 6.837350000 & -5.807277000 & 2.243507000 & $\mathrm{H}$ & 6.827154000 & -5.802991000 & 2.249859000 \\
\hline $\mathrm{H}$ & 6.264431000 & -7.486977000 & 2.127276000 & $\mathrm{H}$ & 6.252820000 & -7.482395000 & 2.137035000 \\
\hline $\mathrm{H}$ & 4.637755000 & 6.426986000 & 2.710760000 & $\mathrm{H}$ & 4.630229000 & 6.427198000 & 2.701767000 \\
\hline $\mathrm{H}$ & 3.323116000 & 6.781065000 & 1.558509000 & $\mathrm{H}$ & 3.315553000 & 6.781084000 & 1.549447000 \\
\hline $\mathrm{H}$ & 4.641804000 & 7.953299000 & 1.785874000 & $\mathrm{H}$ & 4.634053000 & 7.953475000 & 1.776865000 \\
\hline $\mathrm{H}$ & 3.799491000 & 6.766628000 & -0.963762000 & $\mathrm{H}$ & 3.791788000 & 6.766529000 & -0.972777000 \\
\hline $\mathrm{H}$ & 5.441335000 & 6.395018000 & -1.553674000 & $\mathrm{H}$ & 5.433658000 & 6.395094000 & -1.562826000 \\
\hline $\mathrm{H}$ & 5.117006000 & 7.934845000 & -0.712150000 & $\mathrm{H}$ & 5.109129000 & 7.934928000 & -0.721438000 \\
\hline $\mathrm{H}$ & 7.348654000 & 6.015858000 & 0.107739000 & $\mathrm{H}$ & 7.341467000 & 6.017248000 & 0.098632000 \\
\hline $\mathrm{H}$ & 7.016201000 & 6.028585000 & 1.865407000 & $\mathrm{H}$ & 7.009072000 & 6.030183000 & 1.856396000 \\
\hline $\mathrm{H}$ & 6.913001000 & 7.531917000 & 0.924845000 & $\mathrm{H}$ & 6.905144000 & 7.533174000 & 0.915498000 \\
\hline $\mathrm{H}$ & -5.947948000 & 6.224922000 & 1.411576000 & $\mathrm{H}$ & -5.939562000 & 6.227335000 & 1.404815000 \\
\hline $\mathrm{H}$ & -7.265492000 & 5.797899000 & 0.287595000 & $\mathrm{H}$ & -7.256793000 & 5.799294000 & 0.280795000 \\
\hline $\mathrm{H}$ & -6.688914000 & 7.478361000 & 0.379803000 & $\mathrm{H}$ & -6.678771000 & 7.479368000 & 0.370137000 \\
\hline $\mathrm{H}$ & -3.376337000 & 6.974675000 & -1.256053000 & $\mathrm{H}$ & -3.365915000 & 6.970727000 & -1.262978000 \\
\hline $\mathrm{H}$ & -3.681187000 & 6.978795000 & 0.506822000 & $\mathrm{H}$ & -3.671747000 & 6.978580000 & 0.499779000 \\
\hline $\mathrm{H}$ & -4.502314000 & 8.151212000 & -0.544992000 & $\mathrm{H}$ & -4.491744000 & 8.149121000 & -0.554928000 \\
\hline $\mathrm{H}$ & -6.837372000 & 5.807292000 & -2.243448000 & $\mathrm{H}$ & -6.827153000 & 5.802995000 & -2.249850000 \\
\hline $\mathrm{H}$ & -5.223801000 & 6.240552000 & -2.867442000 & $\mathrm{H}$ & -5.212822000 & 6.233476000 & -2.873920000 \\
\hline $\mathrm{H}$ & -6.264456000 & 7.486992000 & -2.127201000 & $\mathrm{H}$ & -6.252821000 & 7.482399000 & -2.137023000 \\
\hline $\mathrm{H}$ & -7.016180000 & -6.028570000 & -1.865479000 & $\mathrm{H}$ & -7.009064000 & -6.030174000 & -1.856429000 \\
\hline $\mathrm{H}$ & -7.348639000 & -6.015873000 & -0.107812000 & $\mathrm{H}$ & -7.341462000 & -6.017252000 & -0.098666000 \\
\hline $\mathrm{H}$ & -6.912978000 & -7.531917000 & -0.924942000 & $\mathrm{H}$ & -6.905136000 & -7.533171000 & -0.915542000 \\
\hline $\mathrm{H}$ & -3.323094000 & -6.781045000 & -1.558581000 & $\mathrm{H}$ & -3.315545000 & -6.781074000 & -1.549478000 \\
\hline $\mathrm{H}$ & -4.637730000 & -6.426952000 & -2.710832000 & $\mathrm{H}$ & -4.630219000 & -6.427183000 & -2.701798000 \\
\hline $\mathrm{H}$ & -4.641778000 & -7.953279000 & -1.785968000 & $\mathrm{H}$ & -4.634043000 & -7.953465000 & -1.776905000 \\
\hline $\mathrm{H}$ & -5.441324000 & -6.395051000 & 1.553600000 & $\mathrm{H}$ & -5.433656000 & -6.395104000 & 1.562793000 \\
\hline $\mathrm{H}$ & -3.799476000 & -6.766645000 & 0.963688000 & $\mathrm{H}$ & -3.791785000 & -6.766534000 & 0.972746000 \\
\hline $\mathrm{H}$ & -5.116986000 & -7.934864000 & 0.712054000 & $\mathrm{H}$ & -5.109124000 & -7.934933000 & 0.721398000 \\
\hline
\end{tabular}


Table S9. DFT optimized ground state $\left(\mathrm{S}_{0}\right)$ and $\mathrm{T}_{1}$ state geometry of $\mathbf{I r I} \mathbf{r}$ ' in cartesian (XYZ) coordinates

\begin{tabular}{|c|c|c|c|c|c|c|c|}
\hline \multicolumn{4}{|c|}{ State $S_{0}$} & \multicolumn{4}{|c|}{ State $T_{1}$} \\
\hline$C$ & -3.320607000 & 2.363840000 & -3.714828000 & $C$ & 3.279638000 & -2.385965000 & -3.714838000 \\
\hline C & -3.396805000 & 2.003855000 & -2.372920000 & C & 3.358519000 & -2.027945000 & -2.372619000 \\
\hline $\mathrm{N}$ & -3.643823000 & 0.713654000 & -2.025374000 & $\mathrm{~N}$ & 3.628170000 & -0.742327000 & -2.025423000 \\
\hline C & -3.795997000 & -0.194231000 & -2.979972000 & C & 3.803140000 & 0.163176000 & -2.978065000 \\
\hline C & -3.728910000 & 0.105435000 & -4.322541000 & C & 3.734526000 & -0.135034000 & -4.320644000 \\
\hline C & -3.487413000 & 1.420564000 & -4.720066000 & C & 3.467745000 & -1.445318000 & -4.718867000 \\
\hline C & -3.231257000 & 2.900115000 & -1.237843000 & C & 3.176774000 & -2.923122000 & 48000 \\
\hline C & -2.940339000 & 4.259266000 & 0000 & C & 53210000 & 77621000 & 00 \\
\hline C & -2 & 4.9 & & C & & & \\
\hline C & & 4.2 & 1.20 & C & 0000 & 7617000 & 9000 \\
\hline C & -3.234280000 & 2.898885000 & 1.238298000 & C & 3.176774000 & -2.923117000 & 1.238160000 \\
\hline C & -3.358885000 & 2.262132000 & -0.000243000 & C & 3.315067000 & -2.290360000 & 04000 \\
\hline$C$ & -3.402910000 & 2.001511000 & 2.372051000 & C & 3.358519000 & -2.027935000 & 2.372627000 \\
\hline C & -3.330243000 & 2.360173000 & 3.714522000 & C & 3.279636000 & -2.385950000 & 3.714847000 \\
\hline C & -3.499892000 & 1.415946000 & 9000 & C & 3.467743000 & -1.445299000 & 72000 \\
\hline C & -3.740584000 & 6000 & 00 & C & 4000 & 5017000 & 1000 \\
\hline C & -3.804122000 & 0000 & & C & 0000 & 188000 & 000 \\
\hline $\mathrm{N}$ & -3. & & & $\mathrm{~N}$ & 00 & 000 & 000 \\
\hline C & -3.420 & $1.7 \mathrm{~s}$ & -6 & C & 000 & 587000 & .000 \\
\hline C & -3.436015000 & 1.786116000 & 000 & C & 8000 & -1.814564000 & 7000 \\
\hline $\mathrm{F}$ & -2.787658000 & 4.963631000 & -2.29 & $\mathrm{~F}$ & 3000 & -4.978542000 & 4000 \\
\hline$F$ & -2.793248000 & 4.961356000 & 8000 & $\mathrm{~F}$ & 2.699434000 & -4.978534000 & 3000 \\
\hline 0 & -2.451763000 & 6.235728000 & 000 & 0 & 2.340791000 & -6.245437000 & 0.000012000 \\
\hline C & -3.538 & 7.107 & & C & 000 & 954000 & 9000 \\
\hline $\mathrm{H}$ & -3.12 & 20000 & -3. & $\mathrm{H}$ & 000 & 173000 & 5000 \\
\hline $\mathrm{H}$ & -3.5 & -1.2 & & $\mathrm{H}$ & & & \\
\hline $\mathrm{H}$ & -3.8 & & & $\mathrm{H}$ & & & 00 \\
\hline Ir & -3.7 & 0.37 & $-0 . c$ & Ir & 000 & 000 & 000 \\
\hline $\mathrm{H}$ & -3.13 & 3.40 & & $\mathrm{H}$ & 6000 & 157000 & 8000 \\
\hline $\mathrm{H}$ & -3.879171000 & $-0.70 c$ & 00 & $\mathrm{H}$ & 0000 & 0.663341000 & 3000 \\
\hline $\mathrm{H}$ & -3.991958000 & -1.226046000 & 2.63 & $\mathrm{H}$ & 4.011469000 & 1.187703000 & 43000 \\
\hline $\mathrm{H}$ & -4.173985000 & 6.975605000 & 0.90 & $\mathrm{H}$ & 0000 & -7.015043000 & 0.900086000 \\
\hline $\mathrm{H}$ & -4.171123000 & 6.976763000 & -0.89 & $\mathrm{H}$ & 000 & 5019000 & 54000 \\
\hline $\mathrm{H}$ & -3.137867000 & 8.13 & & $\mathrm{H}$ & 00 & 000 & -0. \\
\hline$c$ & 3.3 & 00 & & C & & & \\
\hline C & & & & C & & 00 & 00 \\
\hline $\mathrm{N}$ & & & & $\mathrm{N}$ & & & -2 \\
\hline C & 3.7 & & & C & 00 & 1000 & 000 \\
\hline C & 3.728912000 & -0.105425000 & -4.322539000 & C & -3.734525000 & 0.135037000 & -4.320643000 \\
\hline C & 3.487412000 & -1.420553000 & -4.720068000 & C & -3.467745000 & 1.445322000 & -4.718865000 \\
\hline C & 3.231258000 & -2.900113000 & -1.237848000 & C & -3.176776000 & 2.923123000 & -1.238146000 \\
\hline$c$ & 2.940339000 & -4.259263000 & -1.204299000 & C & -2.863211000 & 4.277622000 & -1.205979000 \\
\hline C & 2.785150000 & -4.939775000 & 0.001770000 & C & -2.695833000 & 4.955667000 & 0.000013000 \\
\hline C & 67000 & -4.25 & 1.2 & C & 00 & 000 & 000 \\
\hline C & 3.2 & & & C & & 000 & 1.238162000 \\
\hline C & & -2.26 & -0.0 & C & 000 & 2.290360000 & 000 \\
\hline 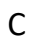 & 3.40 & -2.0 & & C & & 00 & 9000 \\
\hline$c^{2}+2$ & 1000 & -2.3 & & C & 33000 & 2.385947000 & 9000 \\
\hline c & 3.499904000 & -1.415961000 & 78000 & C & -3.467739000 & 1.445295000 & 4.718873000 \\
\hline c & 3.740595000 & -0.101260000 & 4.318921000 & C & -3.734521000 & 0.135013000 & 4.320645000 \\
\hline C & 3.804129000 & 0.197079000 & 2.975893000 & C & -3.803138000 & -0.163190000 & 2.978064000 \\
\hline $\mathrm{N}$ & 3.649246000 & -0.711710000 & 2.022584000 & $\mathrm{~N}$ & -3.628169000 & 0.742317000 & 2.025427000 \\
\hline C & 3.420251000 & -1.792305000 & & C & & 93000 & م 15119900 \\
\hline C & 3.43602900 & & 6.15106 & C & -3.396260000 & 1.814558000 & 6.151209000 \\
\hline $\mathrm{r}$ & 2.787656000 & -4.963625000 & -2.296563000 & $r$ & -2.699436000 & 4.978545000 & -2.298301000 \\
\hline
\end{tabular}




\begin{tabular}{|c|c|c|c|c|c|c|c|}
\hline$F$ & 2.793251000 & -4.961364000 & 2.300107000 & $\mathrm{~F}$ & -2.699432000 & 4.978532000 & 2.298327000 \\
\hline 0 & 2.451762000 & -6.235729000 & 0.002848000 & 0 & -2.340791000 & 6.245437000 & 0.000017000 \\
\hline C & 3.538925000 & -7.107769000 & 0.001658000 & C & -3.413165000 & 7.135954000 & 0.000019000 \\
\hline $\mathrm{H}$ & 3.124264000 & -3.410010000 & -3.976399000 & $\mathrm{H}$ & -3.064890000 & 3.428176000 & -3.977753000 \\
\hline $\mathrm{H}$ & 3.984440000 & 1.223575000 & -2.639943000 & $\mathrm{H}$ & -4.011468000 & -1.187691000 & -2.634849000 \\
\hline H & 3.865274000 & 0.696083000 & -5.059985000 & $\mathrm{H}$ & -3.889661000 & -0.663318000 & -5.057716000 \\
\hline$\| \mathrm{r}$ & 3.715366000 & -0.377076000 & -0.001667000 & Ir & -3.697178000 & 0.407768000 & 0.000001000 \\
\hline $\mathrm{H}$ & 3.134478000 & -3.406084000 & 3.977635000 & $\mathrm{H}$ & -3.064882000 & 3.428154000 & 3.977771000 \\
\hline $\mathrm{H}$ & 3.879187000 & 0.700927000 & 5.055210000 & $\mathrm{H}$ & & -0.663345000 & 5.057713000 \\
\hline $\mathrm{H}$ & $3.9 \mathrm{~s}$ & 1.2 & 2.63 & $\mathrm{H}$ & -4.011 & -1.1 & 3000 \\
\hline $\mathrm{H}$ & 4.173991000 & -6.975610000 & 0.900562 & $\mathrm{H}$ & -4.048 & 7.0150 & 7000 \\
\hline $\mathrm{H}$ & 4.171115000 & -6.976762000 & -0.899441000 & $\mathrm{H}$ & -4.048726000 & 7.015035000 & -0.900052000 \\
\hline $\mathrm{H}$ & 3.137865000 & -8.133578000 & 0.002934000 & $\mathrm{H}$ & -2.994558000 & 8.154668000 & 0.000025000 \\
\hline S & -0.058221000 & 2.079554000 & 0.001493000 & $\mathrm{~s}$ & 0.025987000 & -2.096400000 & 0.000004000 \\
\hline C & 1.805174000 & 0.200080000 & 0.000740000 & C & -1.797440000 & -0.160062000 & 0.000002000 \\
\hline C & 1.603519000 & 1.576968000 & 0.000999000 & C & -1.619992000 & -1.585609000 & 0.000002000 \\
\hline C & 0.552067000 & -0.432819000 & 0.00138 & C & -0.557052000 & 06000 & 2000 \\
\hline C & -0.552067000 & 0.4328 & & C & & & 3000 \\
\hline C & -1.80 & -0.2 & & C & & & 00 \\
\hline c & -1.603 & 74000 & 0.0 & C & 1.6 & 00 & 000 \\
\hline s & 0.058220000 & -2.079560000 & 0.00 & $\mathrm{~s}$ & 87000 & 2.09 & 000 \\
\hline C & 2.725506000 & 2.459300000 & -0.00 & C & -2.740567000 & -2.43837 & 1000 \\
\hline C & -2.725508000 & -2.459304000 & -0.000 & C & 2.7405 & 2.4383 & -0.000005000 \\
\hline $\mathrm{N}$ & -3.913811000 & -1.815006000 & -0.002950000 & $\mathrm{~N}$ & 3.935169000 & 1.773916000 & -0.000005000 \\
\hline C & -5.034927000 & -2.51 & -0.00 & C & & 2.46 & -0.00 \\
\hline C & -5.051328000 & -3.897609000 & -0.00 & C & & & 5000 \\
\hline C & -3.835667000 & -4.5 & -0.0 & C & & & 000 \\
\hline C & -2.66 & -3.8 & -0.0 & C & 00 & & 000 \\
\hline C & 2.6 & 3.8 & -0.0 & C & 304000 & & 5000 \\
\hline C & 3.835658000 & 4.57 & -0.00 & C & -3.878160000 & 713000 & 13000 \\
\hline C & 5.051321000 & 3.897612000 & -0.007854000 & C & -5.093010000 & -3.848815000 & -0.000016000 \\
\hline C & 5.034924000 & 2.513576000 & -0.006379000 & C & -5.057192000 & -2.461964000 & -0.000011000 \\
\hline $\mathrm{N}$ & 3.913811000 & 1.815006000 & -0.002944000 & $\mathrm{~N}$ & -3.935169000 & -1.773916000 & -0.000004000 \\
\hline C & -6.337414000 & -4.653433000 & 0.005265000 & C & 6.382580000 & 4.588 & -0.000021000 \\
\hline C & 6.337402000 & 4.653444000 & 0.00 & C & -6.38 & -4.58 & -0.000023000 \\
\hline $\mathrm{F}$ & 7.36 & 3.86 & -0.1 & $\mathrm{~F}$ & -7. & -3.7 & 00 \\
\hline $\mathrm{F}$ & 6.36 & 5.5 & -0 . & $\mathrm{F}$ & 00 & 00 & 000 \\
\hline $\mathrm{F}$ & 6.5 & & & $\mathrm{~F}$ & & & 000 \\
\hline $\mathrm{F}$ & -6.364946000 & 0000 & -0.9 & $\mathrm{~F}$ & 3582000 & 5.37 & 5000 \\
\hline $\mathrm{F}$ & -6.523281000 & -5.279991000 & 1.146328000 & $\mathrm{~F}$ & 6.498608000 & 5.372093000 & 1.051920000 \\
\hline $\mathrm{F}$ & -7.368383000 & -3.868990000 & -0.179878000 & $\mathrm{~F}$ & 7.414298000 & 3.781828000 & -0.000043000 \\
\hline त & -5.965252000 & -1.922805000 & -0.010387000 & $\mathrm{H}$ & 5.983542000 & 1.864640000 & -0.000010000 \\
\hline H & -3.808878000 & -5.678241000 & -0.008744000 & $\mathrm{H}$ & 3.866400000 & 5.650553000 & -0.000020000 \\
\hline $\mathrm{H}$ & 1.693284000 & 4.366570000 & -0.001321000 & $\mathrm{H}$ & -1.738728000 & -4.369253000 & -0.000003000 \\
\hline $\mathrm{H}$ & 5000 & 00 & -0.0 & $\mathrm{H}$ & -3.86 & -5.65 & -0.0 \\
\hline $\mathrm{H}$ & 5.965251000 & 1.92 & -0.6 & $\mathrm{H}$ & 00 & 640000 & -0.0 \\
\hline $\mathrm{H}$ & -1.693 & -4.3 & & $\mathrm{H}$ & & & 000 \\
\hline , & 56000 & -0.73 & -0.6 & $\mathrm{Cl}$ & -6.139 & & 000 \\
\hline$C$ & -6.168052000 & 0.738993000 & -0.004889000 & $\mathrm{Cl}$ & 6.139061000 & -0.805535000 & 0.000001000 \\
\hline $\mathrm{H}$ & -4.402576000 & 1.657364000 & -6.640886000 & $\mathrm{H}$ & 4.377711000 & -1.681467000 & -6.641941000 \\
\hline $\mathrm{H}$ & -2.712107000 & 1.147661000 & -6.701978000 & $\mathrm{H}$ & 2.689011000 & -1.166011000 & -6.697652000 \\
\hline$\pi$ & -3.115303000 & 2.841111000 & -6.294748000 & $\mathrm{H}$ & 3.086681000 & -2.861764000 & -6.295072000 \\
\hline $\mathrm{H}$ & -4.417658000 & 1.644222000 & 6.639092000 & $\mathrm{H}$ & 4.377739000 & -1.681533000 & 6.641919000 \\
\hline $\mathrm{H}$ & -3.137413000 & 2.836491000 & 6.295339000 & $\mathrm{H}$ & 3.086600000 & -2.861716000 & 6.295084000 \\
\hline ו & -2.724104000 & 1.145035000 & 6.700242000 & $\mathrm{H}$ & 2.689086000 & -1.165929000 & 6.697681000 \\
\hline ד & 4.402582000 & -1.657372000 & -6.640881000 & $\mathrm{H}$ & -4.377739000 & 1.681552000 & -6.641914000 \\
\hline $\mathrm{H}$ & 2.712125000 & -1.147630000 & -6.701985000 & $\mathrm{H}$ & -2.689080000 & 1.165968000 & -6.697673000 \\
\hline $\mathrm{H}$ & 3.115279000 & -2.841090000 & -6.294753000 & $\mathrm{H}$ & -3.086614000 & 2.861749000 & -6.295071000 \\
\hline $\mathrm{H}$ & 3.137476000 & -2.836525000 & 6.295334000 & $\mathrm{H}$ & -4.377721000 & 1.681496000 & 6.641932000 \\
\hline
\end{tabular}




\begin{tabular}{llll|llll}
$\mathrm{H}$ & 2.724076000 & -1.145090000 & 6.700229000 & $\mathrm{H}$ & -3.086620000 & 2.861718000 & 6.295086000
\end{tabular}

$\begin{array}{lllllllll}\text { H } & 4.417656000 & -1.644188000 & 6.639104000 & \text { H } & -2.689053000 & 1.165942000 & 6.697672000\end{array}$

\section{References.}

(1) Daniels, R. E.; Culham, S.; Hunter, M.; Durrant, M. C.; Probert, M. R.; Clegg, W.; Williams, J. A.; Kozhevnikov, V. N. When two are better than one: bright phosphorescence from nonstereogenic dinuclear iridium(III) complexes. Dalton Trans. 2016, 45, 6949-6962.

(2) Bon, J. L.; Feng, D.; Marder, S. R.; Blakey, S. B. A C-H Functionalization Protocol for the Direct Synthesis of Benzobisthiazole Derivatives. J. Org. Chem 2014, 79, 7766-7771.

(3) Frisch, M. J.; Trucks, G. W.; Schlegel, H. B.; Scuseria, G. E.; Robb, M. A.; Cheeseman, J. R.; Scalmani, G.; Barone, V.; Mennucci, B.; Petersson, G. A.; Nakatsuji, H.; Caricato, M.; Li, X.; Hratchian, H. P.; Izmaylov, A. F.; Bloino, J.; Zheng, G.; Sonnenberg, J. L.; Hada, M.; Ehara, M.; Toyota, K.; Fukuda, R.; Hasegawa, J.; Ishida, M.; Nakajima, T.; Honda, Y.; Kitao, O.; Nakai, H.; Vreven, T.; Montgomery Jr., J. A.; Peralta, J. E.; Ogliaro, F.; Bearpark, M. J.; Heyd, J.; Brothers, E. N.; Kudin, K. N.; Staroverov, V. N.; Kobayashi, R.; Normand, J.; Raghavachari, K.; Rendell, A. P.; Burant, J. C.; Iyengar, S. S.; Tomasi, J.; Cossi, M.; Rega, N.; Millam, N. J.; Klene, M.; Knox, J. E.; Cross, J. B.; Bakken, V.; Adamo, C.; Jaramillo, J.; Gomperts, R.; Stratmann, R. E.; Yazyev, O.; Austin, A. J.; Cammi, R.; Pomelli, C.; Ochterski, J. W.; Martin, R. L.; Morokuma, K.; Zakrzewski, V. G.; Voth, G. A.; Salvador, P.; Dannenberg, J. J.; Dapprich, S.; Daniels, A. D.; Farkas, Ö.; Foresman, J. B.; Ortiz, J. V.; Cioslowski, J.; Fox, D. J. Gaussian 09. Gaussian, Inc.: Wallingford, CT, USA, 2009.

(4) Peverati, R.; Truhlar, D. G. Performance of the M11 and M11-L density functionals for calculations of electronic excitation energies by adiabatic time-dependent density functional theory. Phys. Chem. Chem. Phys. 2012, 14, 11363-11370.

(5) Weigend, F.; Ahlrichs, R. Balanced basis sets of split valence, triple zeta valence and quadruple zeta valence quality for $\mathrm{H}$ to Rn: Design and assessment of accuracy. Phys. Chem. Chem. Phys. 2005, 7, 3297-3305.

(6) Cossi, M.; Rega, N.; Scalmani, G.; Barone, V. Energies, structures, and electronic properties of molecules in solution with the C-PCM solvation model. J. Comput. Chem. 2003, 24, 669-681. 